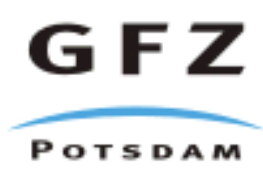

Originally published as:

Trumbull, R. B., Reid, D. L., De Beer, C. H., Romer, R. L. (2007): Magmatism and continental breakup at the west margin of southern Africa: A geochemical comparison of dolerite dikes from NW Namibia and the Western Cape. - South African Journal of Geology, 110, 2-3, 477-502

DOI: 10.2113/gssajg.110.2-3.477. 


\title{
Magmatism and continental breakup at the west margin of southern Africa: A geochemical comparison of dolerite dikes from northwestern Namibia and the Western Cape
}

\author{
Robert B. Trumbull \\ GeoForschungsZentrum Potsdam, Telegrafenberg, 14473 Potsdam, Germany \\ e-mail: bobby@gfz-potsdam.de \\ David L. Reid \\ Dept. of Geological Sciences, University of Cape Town, Rondebosch, South Africa \\ e-mail: dlr@geology.uct.ac.za \\ Coenraad de Beer \\ Council for Geoscience, Bellville, South Africa \\ e-mail: cdebeer@geoscience.org.za \\ David van Acken \\ Institute of Geological Sciences, Free University of Berlin \\ e-mail: david.van.acken@gmx.de \\ Rolf L. Romer \\ GeoForschungsZentrum Potsdam, Telegrafenberg, 14473 Potsdam, Germany: \\ e-mail: romer@gfz-potsdam.de \\ (c) 2007 September Geological Society of South Africa
}

\begin{abstract}
This paper presents a comparative geochemical study of Early Cretaceous mafic dike swarms along the rifted volcanic margin of southern Africa from northwestern Namibia to the Cape Peninsula. These dikes are under study to provide a record of magma compositions and conditions of mantle melting at margin segments with high and low magma flux. Our focus is on localities representing the two ends of the spectrum: the Henties Bay-Outjo dike swarm (HOD) in the high magma-flux Etendeka Province of Namibia, and the False Bay dike swarm from the low-flux margin segment in the Western Cape Province, RSA. At False Bay and other localities examined in the south, dikes are low-Ti, tholeitic dolerites representing a single magma type. Whole-rock MgO contents and olivine compositions in these dikes reach a maximum of 8 weight $\%$ and $\mathrm{FO}_{70}$, respectively. Differentiated dikes $(\mathrm{MgO}=6$ to 2 weight \%) show correlations of trace element and isotopic ratios indicating crustal assimilation, but the least-evolved dikes have uniform incompatible trace element ratios and initial $\mathrm{Sr}-\mathrm{Nd}-\mathrm{Pb}$ isotope compositions $\left({ }^{87} \mathrm{Sr} /{ }^{86} \mathrm{Sr}=0.7056\right.$ to $0.7064, \varepsilon \mathrm{Nd}=$ -1.9 to -2.3 and ${ }^{206} \mathrm{~Pb} /{ }^{204} \mathrm{~Pb}=18.53$ to 18.59 ) suggesting a lithospheric mantle source or a mixture of enriched lithosphere and asthenospheric sources. The dolerites from the HOD swarm in Namibia are mainly low-Ti tholeiites like their counterparts in the south but with much higher Mg-contents (up to 16 weight $\% \mathrm{MgO}$ in whole rock and $\mathrm{FO}_{86-90}$ in olivine). The compositional diversity is also greater in the HOD dikes and at least three magma types are recognized: (1) qz- or ol-normative Tafelberg type, with "spiky" mantle-normalized trace element patterns and initial isotope ratios consistent with a lithospheric mantle source and minor crustal input $\left({ }^{87} \mathrm{Sr} /{ }^{86} \mathrm{Sr}=0.7101\right.$ to $0.7122, \varepsilon \mathrm{Nd}=-2$ to -7 , and ${ }^{206} \mathrm{~Pb} /{ }^{204} \mathrm{~Pb}=18.74$ to 19.10$)$; (2) ol-normative Horingbaai-type with trace element patterns and initial isotope ratios similar to E-MORB $\left({ }^{87} \mathrm{Sr} /{ }^{86} \mathrm{Sr}=0.7037\right.$ to $0.7050, \varepsilon \mathrm{Nd}=+2$ to +6 , and ${ }^{206} \mathrm{~Pb} /{ }^{204} \mathrm{~Pb}=18.00$ to 18.83); and (3) ne-normative magmas, with moderate to high incompatible trace element abundances and intermediate initial isotope ratios $\left({ }^{87} \mathrm{Sr} /{ }^{86} \mathrm{Sr}=0.7055\right.$ to $0.7059, \varepsilon \mathrm{Nd}=0$ to -2 , and ${ }^{206} \mathrm{~Pb} /{ }^{204} \mathrm{~Pb}=18.30$ to 18.61$)$.

The contrast in magmatic intensity between the low- and high-flux margin segments corresponds with systematic compositional differences in contemporary dike swarms. This is supported by geophysical evidence for a change in both the thickness and the average seismic velocity of intruded lower crust at the continent-ocean boundary in the two regions. The greater diversity of magma types and the sharp rise in $\mathrm{MgO}$ contents of dikes in northwestern Namibia (maximum 8 weight \% MgO for False Bay versus 16 weight $\% \mathrm{MgO}$ for $\mathrm{HOD}$ ) suggest that elevated temperatures and active upwelling of a mantle plume caused the enhanced melt production in the north. Dolerite compositions from the False Bay swarm and other localities south of the Orange River are consistent with a passive upwelling model without plume enhancement.
\end{abstract}




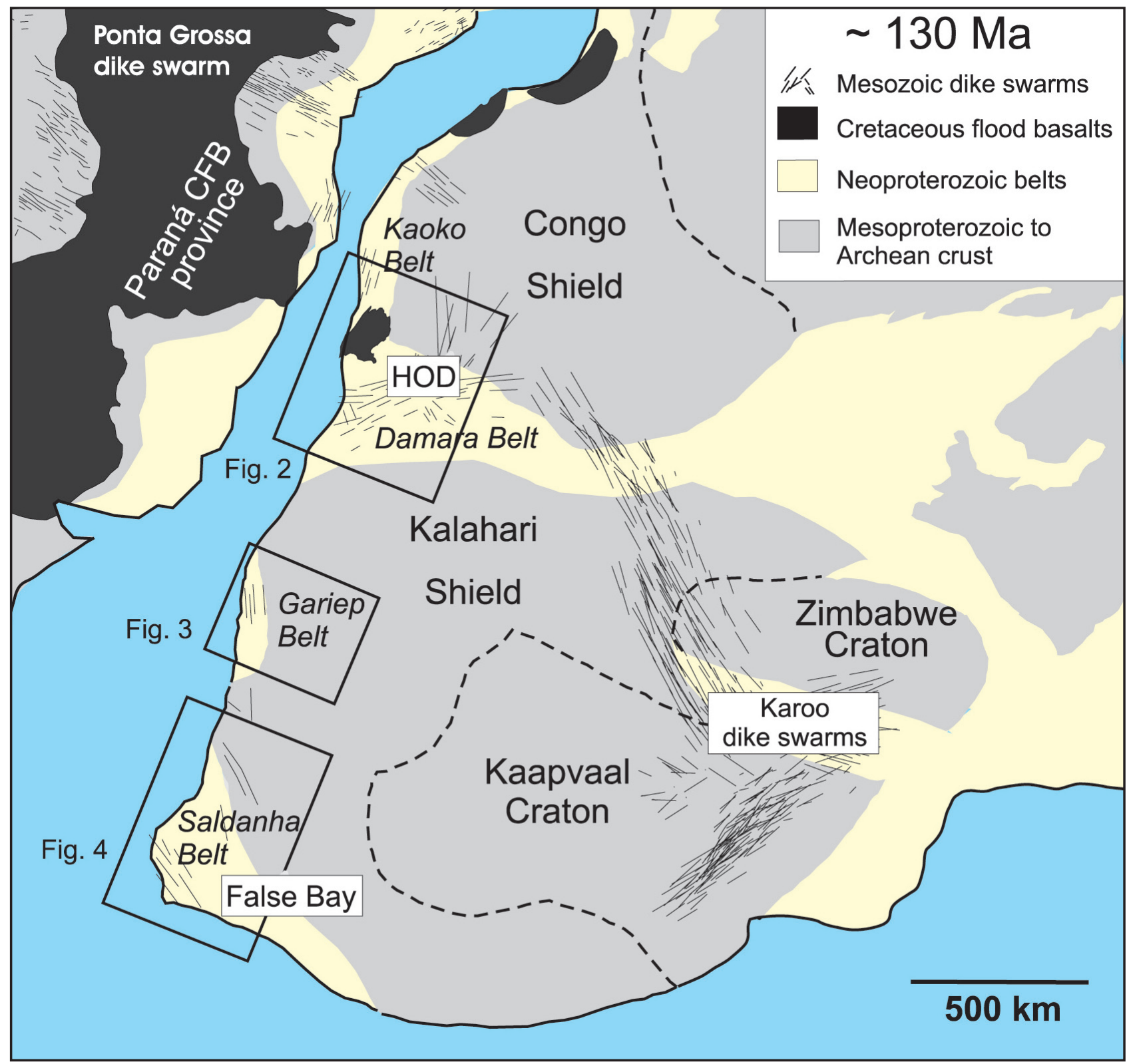

Figure 1. Location sketch map showing a pre-drift reconstruction of southern Africa and south-eastern South America with the approximate boundaries of basement provinces and the distribution of Mesozoic dike swarms and associated continental flood basalts (modified from Trumbull et al., 2004b). Boxes indicate the areas with Early Cretaceous dikes described in this paper.

\section{Introduction}

The Atlantic margin of southern Africa formed in the early Cretaceous $(\sim 130 \mathrm{Ma})$ with the breakup of western Gondwana and it shows the classic features of volcanic rifted margins, with continental flood basalts and central intrusive complexes of the Paraná-Etendeka Large Igneous Province (LIP), as well as seaward-dipping reflector (SDR) sequences and high-velocity underplated igneous crust at the continent-ocean transition (e.g., Mohriak et al., 2002; Trumbull et al., 2002). The ParanáEtendeka LIP is associated with a paired submarine volcanic chain, the Walvis Ridge and Rio Grande Rise, which is considered to mark the track of the Tristan da Cunha hotspot (O'Connor and le Roex, 1992). The association of a hotspot track and a major continental flood basalt province in the South Atlantic is commonly ascribed to a deep-seated thermal plume, analogous to Iceland and the North Atlantic LIP (White and McKenzie, 1989; O'Connor and le Roex, 1992; Saunders et al., 1997). Most of the petrologic and geodynamic attention on magmatism in the South Atlantic has focused on the high magma-flux area of the Paraná-Etendeka LIP, and it is seldom emphasized that Early Cretaceous magmatism was in fact active along the margin as far south as the Cape Peninsula. This is indicated on the one hand by the occurrence of intermittent mafic dikes along the coast, which are the subject of this paper, but also by the near-continuous band of seaward-dipping reflector sequences along the $c a .1800 \mathrm{~km}$ stretch of the margin from the Walvis Ridge to the Cape (Hinz et al., 1999). These SDR sequences are interpreted to represent 
subaerial basalt flows which were submerged and tilted by subsidence of the margin (Hinz, 1981), and it is interesting that their width and thickness do not diminish from north to south (Hinz et al., 1999; S. Neben pers. communication, 2006) despite the strong north to south gradient in intensity of magmatism on land.

Renewed interest in the geodynamic development of the Atlantic margin within the INKABA yeAFRICA program (deWit and Horsfield, 2006) motivated us to undertake a regional study of breakup-related igneous dikes and intrusive complexes along the margin. The focus is on basaltic compositions since these potentially offer petrologic information on the thermal and compositional features of the mantle during breakup. The present paper summarizes the known occurrences of Early Cretaceous dolerite dikes along the margin and presents first results from a comparative study of magma compositions in exemplary localities. Our focus is on two dike swarms at opposite ends of the volcanic margin, the Henties Bay-Outjo dike swarm (HOD) in Namibia (Trumbull et al., 2004b) and the False Bay dike swarm in the Cape Peninsula (Reid et al., 1991).

\section{Geologic framework Basement geology}

The tectonic and magmatic processes leading to formation of the South Atlantic volcanic margins in the Early Cretaceous acted on a series of "Pan-African" (Neoproterozoic to early Cambrian) mobile belts that separate the older cratons and shield areas of Archean and Paleoproterozoic age. From north to south, these Pan-African belts are the Kaoko, Damara, Gariep, and Saldanha (Figure 1). Of relevance in this paper are the Damara and Saldanha belts, which host the HOD and the False Bay dike swarms, respectively. The Damara Belt represents a Neoproterozoic continental platform and rift assemblage between the Congo shield to the north and the Kalahari shield to the south. It consists of medium to high-grade metasediments with mainly semipelitic, carbonate, and graywacke protoliths, and is divided into a number of tectono-lithologic zones separated by major faults, some of which were reactivated in the Mesozoic (see Tankard et al., 1982; Miller, 1983). Partial melting in the high-grade central zone of the Damara belt produced a suite of early Cambrian syn- to post-tectonic granites, the majority of which are S-type (Haack et al., 1982; McDermott et al., 1996; Jung et al., 2000). The Gariep Belt is located at the coast in southern Namibia and northern South Africa, and is host to the Cretaceous Mehlberg dikes (Reid and Rex, 1994). Metasedimentary sequences representing a continental rift and platform setting like the Damara belt are present in the eastern part of the Gariep, and separated by a tectonic suture from western units dominated by oceanic metabasalts (Frimmel and Frank, 1998). The Saldanha Belt is the southernmost PanAfrican belt along the Atlantic margin. It contains lowgrade phyllites, graywackes semipelitic schists and carbonates of the Malmesbury Group which are intruded by early Cambrian granites of the Cape Granite Suite (Rozendaal et al., 1999; Da Silva et al., 2000).

\section{Mesozoic large igneous Provinces}

Large parts of southern Africa and then-adjacent regions of Gondwana in Antarctica and South America were affected by a regional magmatic event in the mid Jurassic (ca. 180 Ma: Duncan et al., 1997). In southern Africa, this event produced the volcanic and intrusive rocks of the Karoo LIP (e.g. Eales et al., 1984; Duncan et al., 1984; Cox, 1988; Marsh et al., 1997). Western outliers of Karoo dolerite sills and basaltic lavas occur in southern Namibia at Marienthal $\left(24.5^{\circ} \mathrm{S}\right)$, near the sites of Early Cretaceous dikes in the western Cape Province described in the present study.

The Early Cretaceous Paraná-Etendeka LIP comprises a bimodal sequence of continental flood basalts (CFB) and silicic volcanics (e.g. Peate, 1997; Marsh et al., 2001), a large number of compositionally diverse central intrusive complexes (Harris, 1995; Milner and le Roex, 1996; Trumbull et al., 2000; 2003; 2004a), and the prominent, mostly mafic dike swarms that are the subject of this paper. Offshore components of this LIP include the paired Walvis Ridge-Rio Grande Rise hotspot track, SDR sequences (Gladczenko et al., 1997; Hinz et al., 1999) and thick, high-velocity lower crustal bodies at the continent-ocean transition (Bauer et al., 2000; Trumbull et al., 2002). The volcanic units of the Parana-Etendeka LIP range in age from 128 to $138 \mathrm{Ma}$ (Renne et al., 1996a; 1996b; Jerram et al., 1999; Marzoli et al., 1999; Kirstein et al., 2001). This age range coincides with that of the Damaraland intrusive complexes in Namibia (125 to 137 Ma: Wigand et al., 2004 and references therein). The onset of CFB magmatism in Namibia is well constrained by an ${ }^{40} \mathrm{Ar}^{39} \mathrm{Ar}$ age of $133 \pm 0.3 \mathrm{Ma}$ obtained by Jerram et al. (1999) from lava flows interbedded with aeolian sandstones at the base of the Etendeka sequence. The oldest magnetic spreading anomaly recognized off Namibia is correlated with M4, which represents an age of $127 \mathrm{Ma}$ (Gladczenko et al., 1997), so the flood volcanics and intrusive complexes formed just prior to continental separation.

The onshore magmatism in the Paraná-Etedeka LIP essentially ended with the onset of Atlantic spreading, extending locally to $120 \mathrm{Ma}$ as suggested by isolated dates from the Paraná province (Peate, 1997; Kirstein et al., 2001). Some 50 million years later, magmatism again affected the region but this time the activity was scattered and small in volume, and magma compositions were highly alkalic. Many of these younger intrusions formed along the margins of the Paraná basin in Brazil and northern Uruguay (Morbidelli et al., 1995; Gibson et al., 1997). On the African margin, Late Cretaceous and Tertiary intrusions are known from Angola (Marzoli et al., 1999), central and southern Namibia (Kurszlaukis et al., 1998; Reid et al., 1990) and in the Cape Province (Moore, 1976; Duncan et al., 1978; McIver, 1981; 


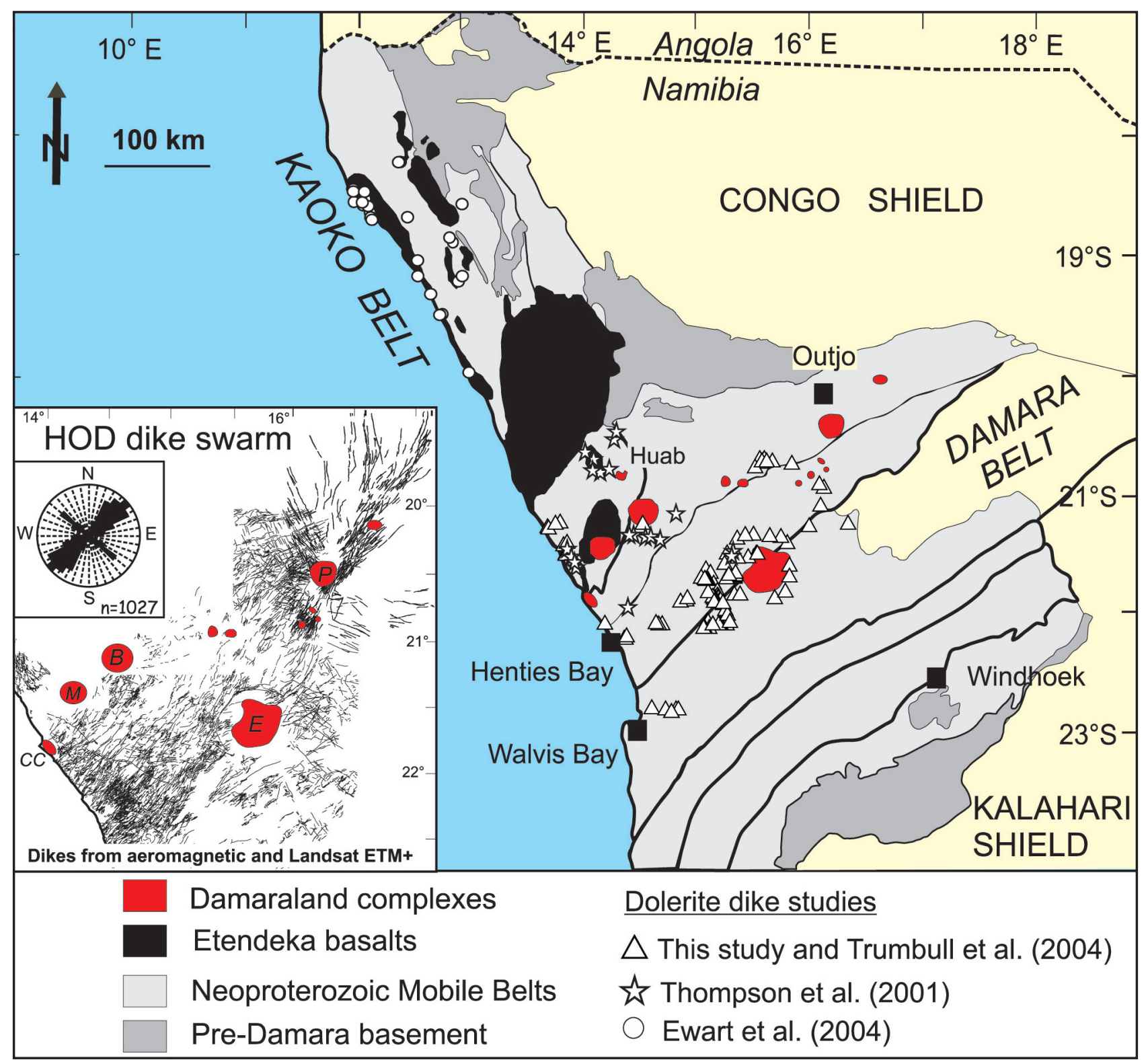

Figure 2. Sketch map with simplified geology of the northern study area with the distribution of Etendeka basalts and Damaraland intrusive complexes ( $\mathrm{E}=$ Erongo, $\mathrm{M}=$ Messum, $\mathrm{B}=$ Brandberg, $\mathrm{P}=$ Paresis, $\mathrm{CC}=$ CapeCross). Inset shows a map of Henties Bay - Outjo dike swarm defined by Trumbull et al., (2004b) on the basis of high-resolution aeromagnetic data and Landsat ETM+ images. An impression of sampling coverage of dikes in this area is given by sample locations from studies by Thompson et al., (2001; 2007, stars), Ewart et al., (2004, circles) and this study (triangles).

Janney et al., 2002). There are reasonable arguments relating the Late Cretaceous alkalic magmatism in Brazil with the Trinidade-Martin Vaz hotspot track (Gibson et al., 1997; Siebel et al., 2000) but post-breakup magmatism on the South African margin is not satisfactorally explained by hotspot models.

\section{Distribution of dolerite dikes}

Dolerite dikes in northwest Namibia have long been recognized and many were included in regional geochemical studies of the CFB province in Namibia and Angola (Erlank et al., 1984; Marzoli et al., 1999; Marsh et al., 2001; Ewart et al., 2004). Many of the dikes are located near the present coast and have coast-parallel strike directions for the most part. The subject of this paper is a prominent inland dike swarm which follows the northeast-southwest structural grain of the Damara Belt and which greatly exceeds the coastal dikes in size and magmatic volume (Figure 2). This is the so-called Henties Bay-Outjo Dike swarm, or HOD (Vietor and Weber, 1999; Trumbull et al., 2004b; Hahne, 2004), which extends at least $400 \mathrm{~km}$ inland from the coast and is thereby comparable in size to the Ponta Grossa swarm in Brazil (Renne et al., 1996a). South of the Kuiseb River, the Namib sand sea precludes systematic dike detection. Local gaps in sand cover at the coast show that dikes are present but none have yet been described from field study. Unfortunately, the high-resolution aeromagnetic surveys that proved effective in mapping buried dikes in the HOD area (Trumbull et al., 2004b), are not freely 


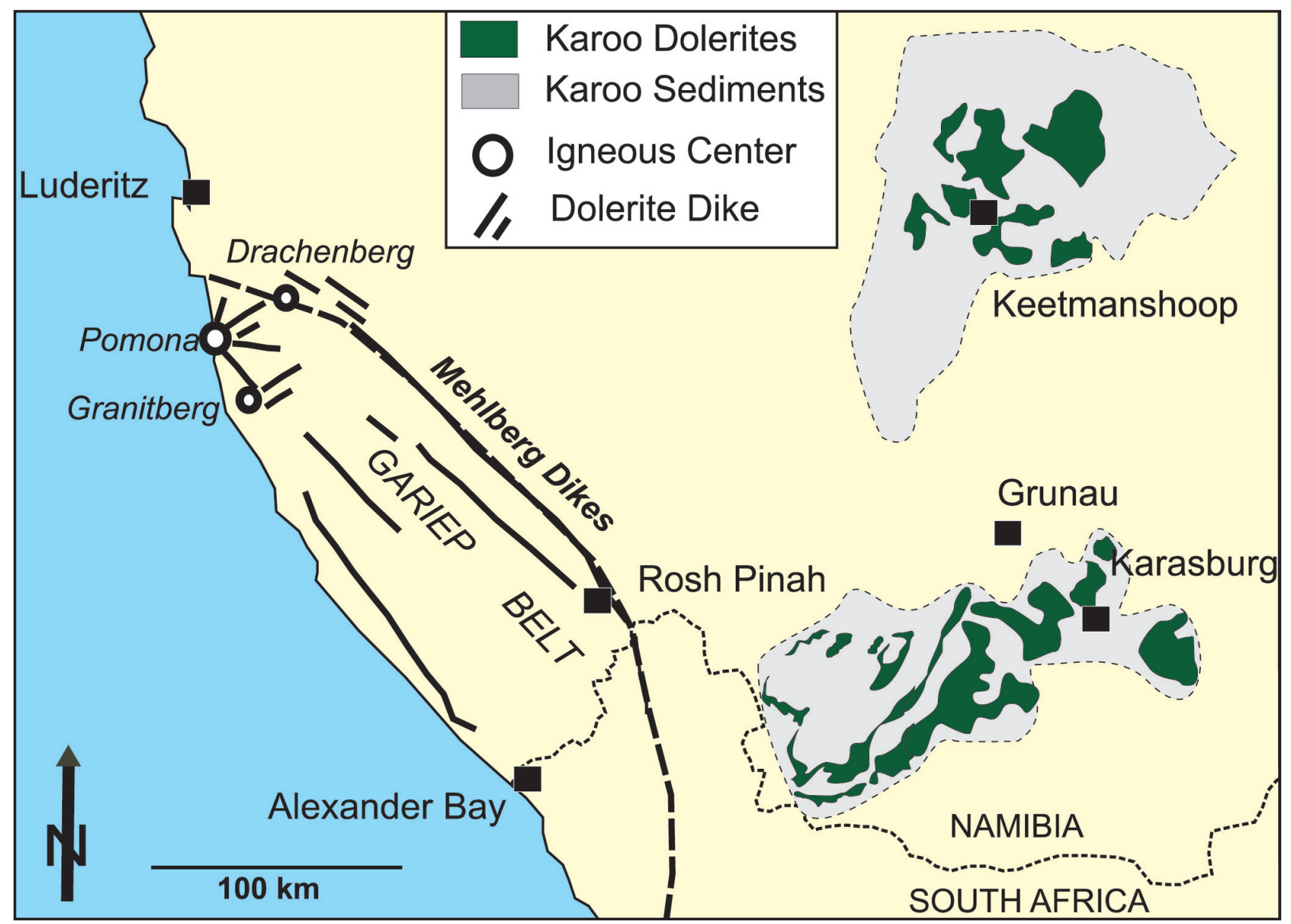

Figure 3. Sketch map of the Lower Orange River border region between Namibia and South Africa, showing the location of the Neoproterozoic Gariep Belt and the distribution of Karoo sediments and dolerite sills. Early Cretaceous intrusives in this area include the Mehlberg dikes (Reid and Rex, 1994), mainly inferred from aeromagnetic surveys, and three Early Cretaceous intrusive centers of the Luderitz alkaline province (after Marsh, 1975; 1976).

available for the coastal sector between Walvis Bay and Luderitz, nor for the Sperrgebeit (diamond restricted area) extending to the Orange River. Our knowledge is thus far based on preliminary aeromagnetic interpretations by Corner (2002). From Luderitz to the Orange River, coastal exposures are only partly obscured by sand cover and a prominent early Cretaceous set of dikes has been identified: the Mehlberg dikes (Reid and Rex, 1994). The largest of these is the Mehlberg dike proper, with an overall width of 10-15 m (Figure 3), which crosses the Orange River at Ochta/Reuning and extends for a considerable distance into the northern Richtersveld (De Villiers and Sohnge, 1959). The Mehlberg dikes develop an en echelon pattern that straddles the regional thrust contact between the Gariep Belt metasediments and the underlying Palaeoproterozoic basement (Von Veh, 1993; Reid and Rex, 1994). Magnetic lineaments that parallel both the coast and the Mehlberg dike in southwest Namibia (Figure 3) are most probably additional members of this swarm. Superimposed on the coastparallel Mehlberg dikes south of Luderitz are small bodies of alkaline felsic rocks (granite, syenite, foyaite) at Pomona, Granitberg and Drachenberg (Figure 3), which Marsh (1975; 1976) interpreted as isolated parts of a larger Cretaceous igneous center that he termed the Luderitz Province. The Luderitz centers are not directly associated with positive magnetic anomalies that would indicate mafic magmas in depth (compare Bauer et al., 2003) but there is a major belt of positive magnetic anomalies just north of the Luderitz Province and intersecting the coast at Luderitz Bay (Corner, 2000; Eberle et al., 2002).

South of the Orange River, several dolerite dikes cutting bedrock of the Neoproterozoic Gariep Belt have been reported from diamond mines along the coast between Alexander Bay and Kleinsee. The most common strike direction is north-northwest to southsoutheast and the widest dike observed has about $30 \mathrm{~m}$ width (C. de Beer, unpublished data). Geological mapping in the Mesoproterozoic Namaqua Province between Kleinsee and the Olifants River has revealed several generations of dikes cutting basement, with strike directions from east-west to north-south (Jansen, 1960; Marais et al., 2002; de Beer et al., 2002). These dikes can be subdivided according to cross-cutting relationships, azimuth and lithology (de Beer et al., 2002). An older suite of dolerite dikes is cut by more felsic varieties (bostonite and quartz porphyry), the latter apparently related to the syenite-granite Koegel Fontein 


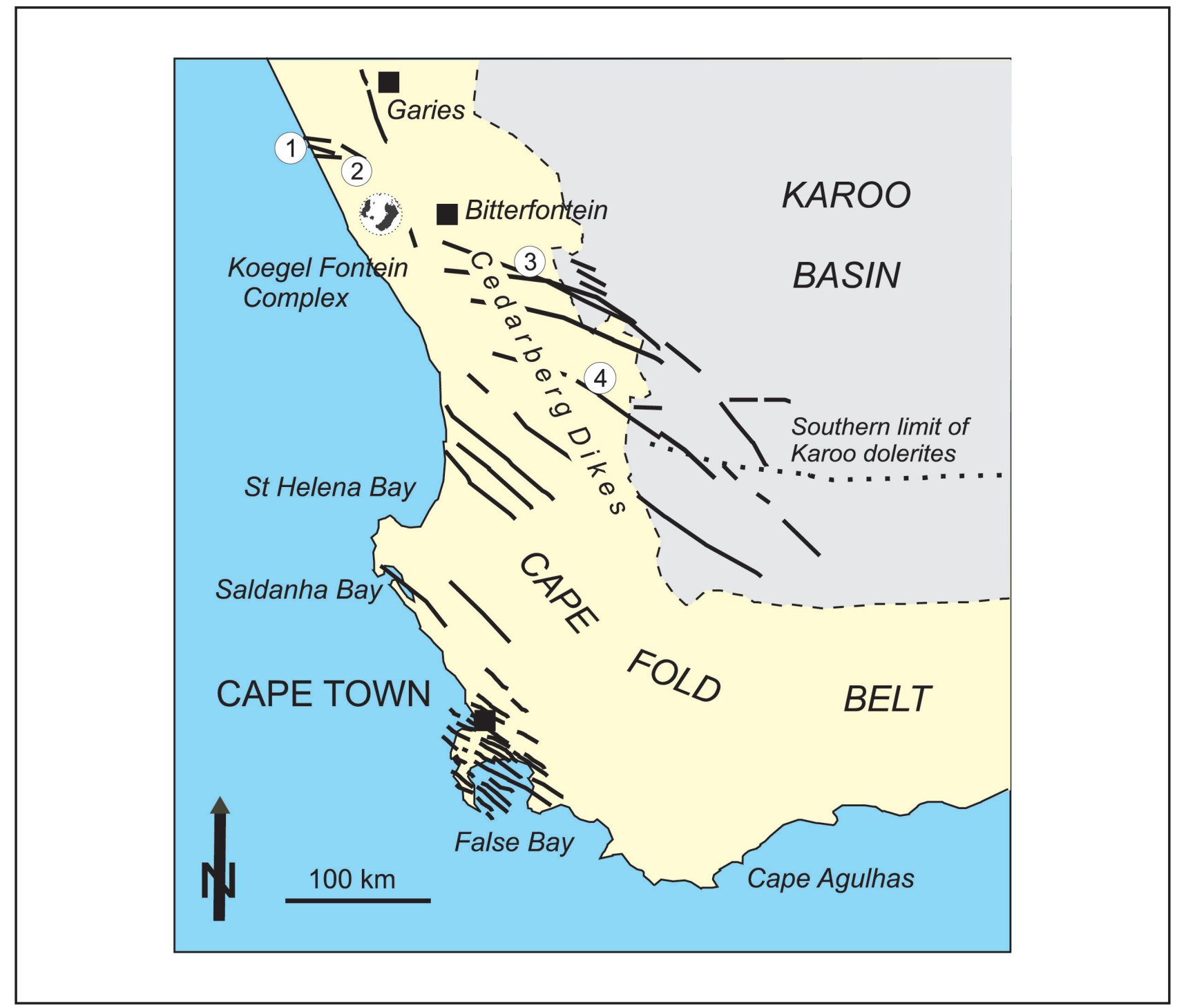

Figure 4. Sketch map showing the distribution of the False Bay and Cedarberg dike swarms in the Western Cape Province of South Africa, based on field and aeromagnetic surveys by Day (1987), Reid (1990), Reeves (2000) and de Beer et al. (2002). Also shown is the Early Cretaceous Koegel Fontein syenite-granite complex (de Beer et al., 2002) which is associated with mainly felsic and alkaline dikes. The numbered circles refer to sample locations as follows (see Table 2): 1 and $2=$ Garies subswarm; $3=$ Knersvlakte dikes, $4=$ Doring-Tanqua dikes.

intrusive center (Figure 4) of Early Cretaceous age (de Beer et al., 2002). Still younger (Tertiary), minor intrusions in this area consist of numerous plugs and discontinuous dikes of alkaline mafic to intermediate compositions (McIver, 1981; Moore and Verwoerd, 1985; de Beer et al., 2002). Farther inland in the western Cape, dolerite dikes are more numerous than at the coast and they follow a fairly consistent northwest to southeast trend (Figure 4). In the northern part of this region, termed the Cedarberg area after Hunter and Reid (1987), the dikes occur in widely spaced en-echelon clusters (Figure 4). Although not radiometrically dated, the Cedarberg dikes are assumed to be of post-Karoo age because they cut dolerite sills that form the western edge of the Karoo igneous province at the escarpment (Chevallier and Woodford, 1999). The presence of major dolerite dikes under thick sand cover between Lamberts Bay and St. Helena Bay is suggested by recent airborne magnetic surveys (C. de Beer, unpublished data). Most of these magnetic lineaments strike west-northwest to east-southeast.

Finally, at the southern tip of the continent is a fairly intense concentration of dikes cutting through the Cape Peninsula, which has been termed the False Bay swarm (Day, 1987). The False Bay dolerite dikes were dated at $132 \pm 6$ Ma by K-Ar (Reid et al., 1991), and a supporting ${ }^{40} \mathrm{Ar}^{39} \mathrm{Ar}$ plagioclase age of $131.3 \pm 1.3 \mathrm{Ma}$ was reported by Stewart et al. (1996).

\section{Field and petrographic descriptions Henties Bay - Outjo Dike swarm}

The Henties Bay-Outjo Dike swarm (Figure 2) contains well over one thousand individual dikes as shown by the interpretation of Landsat ETM+ images and highresolution aeromagnetic data by Hahne (2004) and Trumbull et al. (2004b). The problem of establishing a 
statistically robust estimate of intrusion ages for a dike swarm of this magnitude is apparent, but an Early Cretaceous age is very likely for the great majority of them. Existing K-Ar age determinations of the dikes fall mostly in the range of $\sim 116$ to $\sim 143 \mathrm{Ma}$ (Siedner and Mitchell, 1976; Erlank et al., 1984; Hunter and Reid, 1987), and a number of ${ }^{40} \mathrm{Ar} /{ }^{39} \mathrm{Ar}$ results confirm the Early Cretaceous age. For example, Erlank et al. (1984) reported 125-130 Ma for coastal dikes near Horingbaai, gabbroic sills from the Huab area of the Etendeka province yieded ages of $132 \pm 0.7 \mathrm{Ma}$ (Renne et al., 1996a) and $130.5 \pm 0.8 \mathrm{Ma}$ (Kirstein et al., 2001), and Wigand et al. (2004) determined an age of $132 \pm 1 \mathrm{Ma}$ for basanite-tephrite plugs intruding the Erongo complex. On the other hand, an important discovery of younger intrusions within the HOD was made by Whitehead et al. (2002) who reported a whole-rock ${ }^{40} \mathrm{Ar} /{ }^{39} \mathrm{Ar}$ age of $\sim 80 \mathrm{Ma}$ for nephelinite plugs near Swakopmund.

In coastal exposures north of the HOD, dolerite dikes have mainly coast-parallel strike directions and many of them are syn-tectonic with listric normal faults that offset Etendeka lavas (e.g., Ewart et al., 2004). Coast-parallel dikes are also common within the HOD, but the great majority of dikes strike northeast to southwest, along the structural grain of the Damara Belt (see inset to Figure 2). Where the two trends occur together, detailed examination of satellite images and aeromagnetic maps reveals mutual cross-cutting relations (Hahne, 2004). The dominant northeast to southwest strike of HOD dikes indicates the influence of Damara Belt structures at a regional scale, but locally the dikes commonly crosscut basement foliations and lithologic contacts. The discordance of dike orientation and Damara Belt structures is strongest at the northeastern end of the dike swarm, where dikes leave the Damara Belt altogether and cut across the boundary of the Congo shield (inset, Figure 2). Estimates of the frequency of diking (number of dikes traversed per kilometer perpendicular to strike) from 8 ground traverses of 4 to $7 \mathrm{~km}$ length each, yielded an average of 6 dikes per $\mathrm{km}$ (Hahne, 2004). The measured dikes range in thickness from about $1.5 \mathrm{~m}$ to $45 \mathrm{~m}$ (average $3 \mathrm{~m}$ for 210 measurements) and less than 10\% of the dikes have widths exceeding $5 \mathrm{~m}$. A few doleritic intrusions in the central region of the HOD are up to 500m wide (e.g. Okombahe, Schwarze Spitzkoppe) and they appear from their aeromagnetic signatures to be elongate stocks rather than thick, tabular dikes (Hahne, 2004).

Most of the dolerite dikes from the HOD are holocrystalline. Depending on dike thickness, they are variably fine grained, with exceptional coarsegrained varieties in central parts of thick dikes and in the stock-like intrusions. Typical textures are intersertal to subophitic, with plagioclase, clinopyroxene and olivine being the main mineral phases. Common minor minerals include very fine opaque oxides in the groundmass and acicular apatite. Secondary minerals include sericite in altered plagioclase and biotite, which occurs locally rimming oxide grains or in association with pyroxenes. Many dikes are amygdaloidal, sometimes strongly so at the margins, the open spaces are filled partially or completely by chalcedony and/or carbonate. Finally, evidence for partial melting of the Damara country rocks and back-veining at the margins of thick dikes was noted at several locations.

\section{The False Bay dikes}

The False Bay dikes follow a northwest to southeasterly to west-northwest to east-southeasterly striking series of faults and joint systems that are developed in the Palaeozoic Cape Supergroup and underlying Neoproterozoic (Saldanha Belt) basement. The dikes strike parallel to the inferred continental margin that stretches from Cape Agulhas to St. Helena Bay (Figure 4). Judging from the shoreline outcrops, dike thicknesses vary from a maximum of about $40 \mathrm{~m}$ down to about 1 meter. Whereas outcrop is limited, the regional aero- and marine magnetic patterns (Day, 1987) reveal the presence of a major swarm covering an area at least $100 \mathrm{~km}$ in strike length (northwest to southeast) and more than $70 \mathrm{~km}$ width. The frequency of diking in the False Bay swarm cannot be directly compared with that of the HOD swarm in Namibia because of differences in the quality of exposure and of available magnetic data, but the False Bay swarm clearly represents a local maximum of dike density at the southern margin.

Textural variations in the dikes depend on thickness, with chilled margins passing into medium grained sub-ophitic dolerites in the wider intrusions. Most dikes are aphyric with occasional development of microporphyritic margins bearing plagioclase and olivine. Holocrystalline dikes typically display assemblages of plagioclase and augite, intergrown with minor olivine and set in a discontinuous matrix and dark groundmass rich in microlites of magnetite. Filled vesicles are not uncommon, indicating a relatively shallow depth of emplacement. Several dikes contain enclaves of granitic country rock, often disaggregated into irregular xenocrysts of feldspar or quartz, the latter usually rimmed with reaction pyroxene. Partial melting of the country rock to form felsic net veins has been observed in several localities (e.g. Reid, 1990; Walker, 1956).

\section{Other localities south of the Orange River}

Hunter and Reid (1987) and Reeves (2000) referred to dikes in the region between the Richtersveld and the Cape as the Cedarberg swarm and this name is retained here (Figure 4). The 1:250,000 scale geological maps by Marais et al. (2002) and de Beer et al. (2002) delineate a regional dolerite swarm which is cut by a more restricted group of felsic and alkaline dikes, the latter being spatially associated with the Koegel Fontein intrusive complex. Petrologic and geochemical work on dikes in this region is ongoing and the first results are reported 
Table 1A. Selected geochemical and isotopic analyses for dolerite dikes from the Henties Bay - Outjo dike swarm (HOD).

\begin{tabular}{|c|c|c|c|c|c|c|c|c|c|c|c|c|}
\hline $\begin{array}{l}\text { Sample } \\
\text { Magma type }\end{array}$ & $\begin{array}{r}346 \\
\text { Tafelberg } \\
\end{array}$ & $\begin{array}{r}374 \\
\text { Tafelberg } \\
\end{array}$ & $\begin{array}{r}377 \\
\text { Tafelberg } \\
\end{array}$ & $\begin{array}{r}158 \mathrm{C} \\
\text { Tafelberg } \\
\end{array}$ & $\begin{array}{r}160 \mathrm{C} \\
\text { Tafelberg } \\
\end{array}$ & $\begin{array}{r}\text { Q16 } \\
\text { Tafelberg } \\
\end{array}$ & $\begin{array}{r}\text { Q7 } \\
\text { Tafelberg } \\
\end{array}$ & $\begin{array}{r}\text { RD324 } \\
\text { Tafelberg } \\
\end{array}$ & $\begin{array}{r}\text { RD328A } \\
\text { Tafelberg } \\
\end{array}$ & $\begin{array}{r}\text { RD336 } \\
\text { Tafelberg } \\
\end{array}$ & \multicolumn{2}{|c|}{$\begin{array}{r}\text { RD360B RD-G199 } \\
\text { Tafelberg Tafelberg }\end{array}$} \\
\hline S Latitude & 21.39 & 22.66 & 22.66 & 21.95 & 21.95 & 22.05 & 21.38 & 21.75 & 21.7 & 21.36 & 21.42 & 21.56 \\
\hline E Longitude & 15.51 & 14.79 & 14.90 & 15.32 & 15.26 & 14.45 & 15.42 & 15.4 & 15.37 & 15.61 & 15.9 & 15.38 \\
\hline $\mathrm{SiO}_{2}$ & 47.8 & 49.4 & 47.7 & 49.1 & 48.2 & 44.8 & 47.4 & 49.3 & 49.5 & 49.7 & 49.5 & 47.9 \\
\hline $\mathrm{TiO}_{2}$ & 1.81 & 1.08 & 0.68 & 1.03 & 0.9 & 0.51 & 1.01 & 0.71 & 0.94 & 1.39 & 0.94 & 0.85 \\
\hline $\mathrm{Al}_{2} \mathrm{O}_{3}$ & 14.8 & 13.7 & 13.0 & 15.7 & 12.8 & 10.5 & 14.6 & 16.6 & 17.0 & 15.9 & 17.1 & 12.5 \\
\hline $\mathrm{FeO}$ & 12.0 & 9.69 & 9.33 & 9.18 & 10.1 & 10.1 & 12.7 & 8.4 & 9.1 & 11.1 & 9.82 & 10.3 \\
\hline $\mathrm{MnO}$ & 0.18 & 0.17 & 0.17 & 0.17 & 0.17 & 0.17 & 0.19 & 0.16 & 0.17 & 0.19 & 0.17 & 0.17 \\
\hline $\mathrm{MgO}$ & 7.32 & 10.3 & 13.8 & 7.61 & 12.7 & 21.3 & 10.8 & 7.6 & 6.31 & 5.41 & 6.08 & 13.3 \\
\hline $\mathrm{CaO}$ & 9.18 & 9.9 & 10.6 & 11.9 & 9.52 & 8.36 & 7.65 & 12.6 & 11.8 & 9.98 & 11.4 & 9.13 \\
\hline $\mathrm{Na}_{2} \mathrm{O}$ & 2.85 & 1.94 & 1.42 & 2.03 & 1.58 & 1.25 & 2.28 & 2.13 & 2.4 & 2.6 & 2.33 & 1.4 \\
\hline $\mathrm{K}_{2} \mathrm{O}$ & 0.88 & 0.96 & 0.52 & 0.49 & 0.55 & 0.24 & 1.01 & 0.36 & 0.47 & 0.9 & 0.52 & 0.38 \\
\hline $\mathrm{P}_{2} \mathrm{O}_{5}$ & 0.23 & 0.14 & 0.09 & 0.11 & 0.09 & 0.06 & 0.15 & 0.07 & 0.09 & 0.14 & 0.08 & 0.09 \\
\hline $\mathrm{H}_{2} \mathrm{O}$ & 0.87 & 1.31 & 1.73 & 1.33 & 1.98 & 1.08 & 0.57 & 1.07 & 1.27 & 1.04 & 1.19 & 2.52 \\
\hline $\mathrm{CO}_{2}$ & 1.15 & 0.63 & 0.21 & 0.08 & 0.12 & 0.49 & 0.1 & 0.09 & 0.07 & 0.43 & 0.08 & 0.33 \\
\hline Total & 99.0 & 99.2 & 99.2 & 98.7 & 98.7 & 98.9 & 98.5 & 99.1 & 99.1 & 98.8 & 99.3 & 99.0 \\
\hline $\mathrm{Cr}$ & 190 & 602 & 1300 & 247 & 1300 & 2090 & 110 & 147 & 70 & 36 & 62 & 74 \\
\hline $\mathrm{Ni}$ & 77 & 264 & 469 & 70 & 366 & 968 & 195 & 71 & 53 & 40 & 153 & 56 \\
\hline $\mathrm{Rb}$ & 23 & 27 & 19 & 16 & 16 & $<10$ & 16 & $<10$ & 18 & 24 & 19 & 20 \\
\hline $\mathrm{Sr}$ & 324 & 162 & 116 & 242 & 142 & 68 & 205 & 228 & 248 & 244 & 192 & 245 \\
\hline $\mathrm{Ba}$ & 380 & 248 & 96 & 113 & 168 & 48 & 355 & 76 & 78 & 158 & 151 & 104 \\
\hline $\mathrm{Zn}$ & 93 & 77 & 268 & 71 & 72 & 56 & 95 & 350 & 62 & 88 & 33 & 87 \\
\hline $\mathrm{Zr}$ & 141 & 115 & 69 & 81 & 87 & 42 & 132 & 52 & 63 & 101 & 81 & 61 \\
\hline $\mathrm{Nb}$ & 9.3 & 8.2 & 4 & 4.8 & 4.4 & 6.4 & 9 & 5 & 4.1 & 7.6 & 5.9 & 3 \\
\hline Y & 25 & 26 & 22 & 18 & 19 & 17 & 28 & 20 & 19 & 29 & 19 & 17 \\
\hline $\mathrm{V}$ & 345 & 254 & 249 & 341 & 273 & 208 & 145 & 318 & 390 & 408 & 312 & n.d \\
\hline $\mathrm{Cs}$ & 2.2 & 3.2 & 2.4 & 7.9 & 22 & 1.3 & 0.17 & 1.8 & n.d. & n.d. & n.d. & 1.3 \\
\hline $\mathrm{Sc}$ & 23 & 32 & 33 & 35 & 33 & 35 & 18 & n.d. & 39 & 32 & 25 & 35 \\
\hline Co & 56 & 53 & 63 & 45 & 65 & 55 & 74 & n.d. & 43 & 43 & 82 & 44 \\
\hline $\mathrm{Cu}$ & 78 & 93 & 95 & 62 & 111 & 109 & 50 & n.d. & 68 & 112 & 78 & 99 \\
\hline $\mathrm{Ga}$ & $<10$ & 16 & 14 & 19 & 16 & 20 & 18 & n.d. & 21 & 22 & 18 & 22 \\
\hline $\mathrm{La}$ & 15.5 & 17 & 8 & 11 & 9.7 & 14 & 18 & 5.5 & 7 & 13 & 10 & 8.4 \\
\hline $\mathrm{Ce}$ & 35.9 & 33 & 17 & 24 & 20 & 29 & 36 & 12 & 15 & 28 & 21 & 18 \\
\hline $\mathrm{Pr}$ & 4.9 & 4.5 & 2.2 & 3.2 & 3 & 4.1 & 4.8 & 1.7 & 2.1 & 3.8 & 2.9 & 2.4 \\
\hline $\mathrm{Nd}$ & 21.7 & 18 & 9.2 & 13 & 12 & 17 & 19 & 7.7 & 9 & 16 & 13 & 10 \\
\hline $\mathrm{Sm}$ & 5.1 & 4.1 & 2.4 & 3.4 & 3 & 4.2 & 4.3 & 2.1 & 2.6 & 4.2 & 3.3 & 2.7 \\
\hline $\mathrm{Eu}$ & 1.6 & 1.2 & 0.68 & 1.1 & 0.93 & 1.3 & 1.3 & 0.92 & 1.2 & 1.5 & 1.2 & 1.1 \\
\hline Gd & 5.1 & 4.2 & 2.8 & 3.5 & 3.3 & 4.5 & 4.5 & 2.6 & 3.2 & 4.9 & 3.9 & 3.2 \\
\hline $\mathrm{Tb}$ & 0.77 & 0.76 & n.d. & n.d. & 0.63 & n.d. & 0.79 & 0.43 & 0.5 & 0.78 & 0.61 & 0.53 \\
\hline Dy & 4.6 & 4.2 & 3.2 & 3.5 & 3.6 & 4.6 & 4.5 & 2.8 & 3.2 & 4.8 & 3.8 & 3.5 \\
\hline Ho & 0.90 & 0.84 & 0.67 & 0.76 & 0.7 & 0.9 & 0.89 & 0.56 & 0.65 & 0.99 & 0.78 & 0.72 \\
\hline $\mathrm{Er}$ & 2.5 & 2.5 & 2.0 & 2.2 & 2.2 & 2.8 & 2.7 & 1.6 & 1.9 & 2.9 & 2.3 & 2.1 \\
\hline $\mathrm{Tm}$ & 0.33 & 0.36 & 0.30 & 0.31 & 0.30 & 0.39 & 0.39 & 0.23 & 0.27 & 0.41 & 0.33 & 0.3 \\
\hline $\mathrm{Yb}$ & 2.1 & 2.4 & 1.9 & 2.0 & 2.0 & 2.6 & 2.5 & 1.5 & 1.7 & 2.6 & 2.1 & 1.9 \\
\hline $\mathrm{Lu}$ & 0.3 & 0.36 & 0.29 & 0.3 & 0.3 & 0.39 & 0.37 & 0.22 & 0.27 & 0.41 & 0.34 & 0.28 \\
\hline $\mathrm{Hf}$ & 3.7 & n.d. & n.d. & n.d. & n.d. & n.d. & n.d. & 1.3 & 1.6 & 2.8 & 2.2 & 1.7 \\
\hline $\mathrm{Ta}$ & 0.5 & 0.54 & 0.48 & 0.48 & 0.32 & 0.87 & 0.59 & n.d. & 0.25 & 0.61 & 0.4 & 0.29 \\
\hline Th & 1.6 & 3.3 & 2.4 & 2.0 & 1.6 & 2.5 & 2.6 & 1.3 & 1.9 & 3.9 & 2.9 & 2.1 \\
\hline $\mathrm{U}$ & 0.37 & 0.45 & 0.62 & 0.44 & 0.24 & 0.52 & 0.41 & 0.4 & 0.6 & 1.2 & 0.8 & 0.6 \\
\hline $\mathrm{Pb}$ & 3.3 & 4.8 & 2.9 & 2.8 & 2.6 & 4.5 & 5 & 2.4 & 3.2 & 5.7 & 4.2 & 3.2 \\
\hline$\underline{\mathrm{Li}}$ & 12 & 8.6 & 14 & 20 & 20 & 10 & 7.7 & n.d. & n.d. & n.d. & n.d. & n.d. \\
\hline${ }^{87} \mathrm{Sr} /{ }^{86} \mathrm{Sr}$ mea & isured & & $.713035(14)$ & $0.710567(11) 0$ & $.711569(7)$ & & & $.710280(5)$ & $0.710598(9)$ & $0.711507(7)$ & $0.710917(7)$ & \\
\hline${ }^{87} \mathrm{Sr} /{ }^{86} \mathrm{Sr}(130$ & $\mathrm{Ma})$ & & 0.71217 & 0.71023 & 0.71010 & & & 0.71004 & 0.71014 & 0.71085 & 0.71038 & \\
\hline${ }^{143} \mathrm{Nd} /{ }^{144} \mathrm{Nd}$ & measured & & $.512299(4)$ & $0.512538(5) 0$ & $.512284(5)$ & & & $.512570(7)$ & $0.512550(4)$ & $0.512495(7)$ & $0.512518(5)$ & \\
\hline${ }^{143} \mathrm{Nd} /{ }^{144} \mathrm{Nd}$ & (130 Ma) & & 0.51217 & 0.51241 & 0.51216 & & & 0.51243 & 0.51240 & 0.51236 & 0.51239 & \\
\hline$\varepsilon \mathrm{Nd}(130 \mathrm{Ma})$ & & & -6.0 & -1.3 & -6.2 & & & -0.8 & -1.4 & -2.2 & -1.6 & \\
\hline${ }^{206} \mathrm{~Pb} /{ }^{204} \mathrm{~Pb} \mathrm{n}$ & neasured & & 19.377 & 18.942 & 18.497 & & & 19.220 & 19.230 & 19.210 & 19.252 & \\
\hline${ }^{207} \mathrm{~Pb} /{ }^{204} \mathrm{~Pb} \mathrm{n}$ & neasured & & 15.741 & 15.678 & 15.712 & & & 15.697 & 15.704 & 15.715 & 15.711 & \\
\hline${ }^{208} \mathrm{~Pb} /{ }^{204} \mathrm{~Pb} \mathrm{n}$ & neasured & & 39.588 & 39.122 & 39.104 & & & 39.081 & 39.106 & 39.169 & 39.192 & \\
\hline${ }^{206} \mathrm{~Pb} /{ }^{204} \mathrm{~Pb}$ & $(130 \mathrm{Ma})$ & & 19.09 & 18.74 & 18.38 & & & 18.99 & 18.95 & 18.89 & 18.99 & \\
\hline${ }^{207} \mathrm{~Pb} /{ }^{204} \mathrm{~Pb}$ & (130 Ma) & & 15.73 & 15.67 & 15.71 & & & 15.69 & 15.69 & 15.70 & 15.70 & \\
\hline${ }^{208} \mathrm{~Pb} /{ }^{204} \mathrm{~Pb}($ & $130 \mathrm{Ma})$ & & 39.23 & 38.81 & 38.84 & & & 38.83 & 38.82 & 38.84 & 38.88 & \\
\hline
\end{tabular}

Sample locations in decimal degrees, based on the WG84 datum. Analyses on whole-rock powders at the GeoForschungsZentrum Potsdam.

Major elements by X-ray fluorescence (Phillips PW2400, Rh tube) on fused discs, FeO is total Fe. $\mathrm{H}_{2} \mathrm{O}$ and $\mathrm{CO}_{2}$ by infrared spectrometry (Leco RC-412)

First block of trace elements ( $\mathrm{Cr}$ to V) were analyzed by XRF, the second block by ICP-MS and ICP-OES for the REE, see appendix for details. n.d. = not determined.

Measurement errors for $\mathrm{Sr}$ and $\mathrm{Nd}$ isotope ratios in parenthesis refer to last digits $(2 \sigma)$.

Accuracy and precision for $\mathrm{Pb}$ isotopes are better than $0.1 \%$ at the 2 sigma level, see appendix for analytical details. 
Table 1B. Selected geochemical and isotopic analyses for dolerite dikes from the Henties Bay - Outjo dike swarm (HOD).

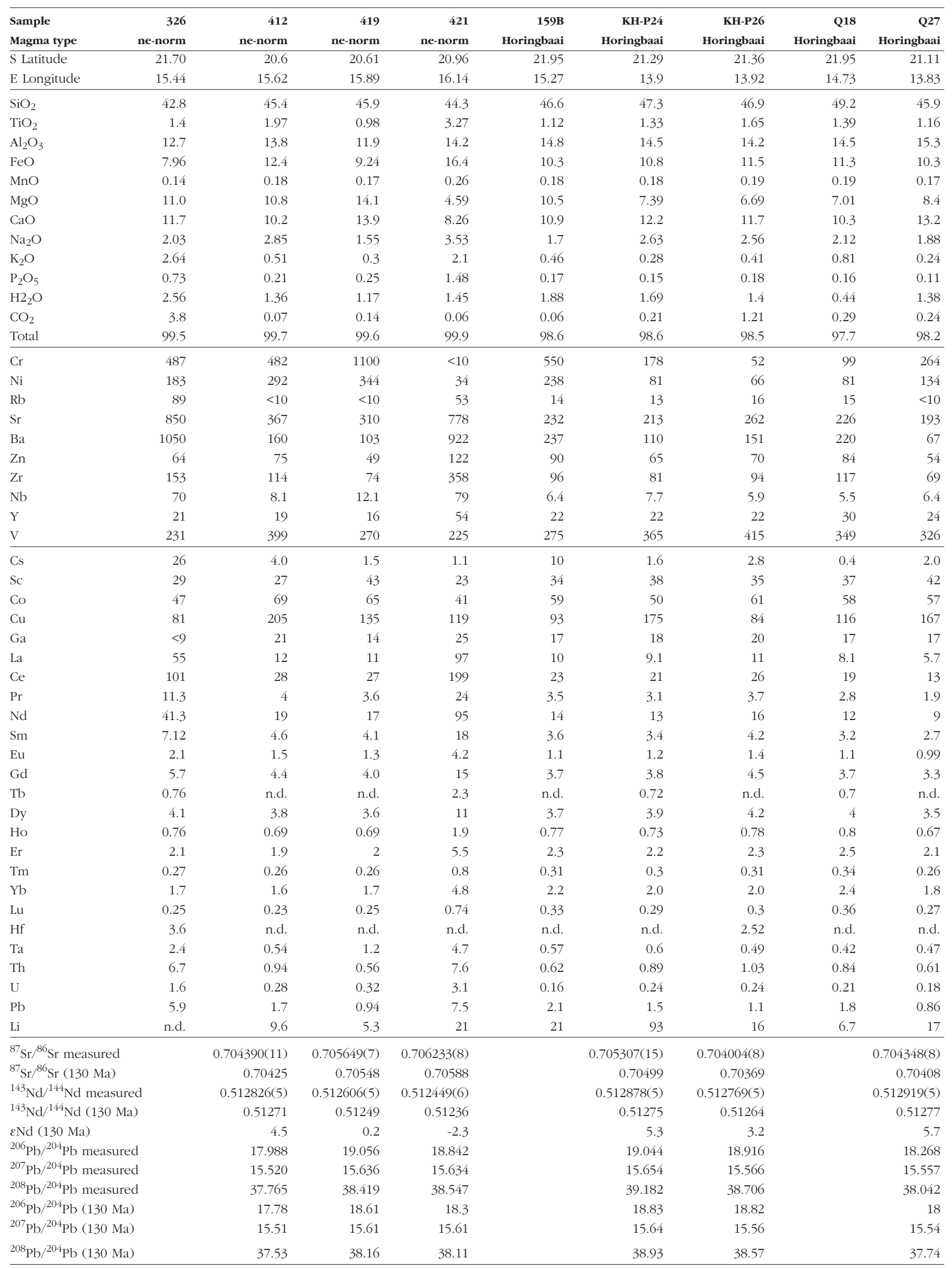

Sample locations in decimal degrees, based on the WG84 datum. Analyses on whole-rock powders at the GeoForschungsZentrum Potsdam.

Major elements by X-ray fluorescence (Phillips PW2400, Rh tube) on fused discs, FeO is total Fe. $\mathrm{H}_{2} \mathrm{O}$ and $\mathrm{CO}_{2}$ by infrared spectrometry (Leco RC-412)

First block of trace elements ( $\mathrm{Cr}$ to V) were analyzed by XRF, the second block by ICP-MS and ICP-OES for the REE, see appendix for details. n.d. $=$ not determined.

Measurement errors for $\mathrm{Sr}$ and $\mathrm{Nd}$ isotope ratios in parenthesis refer to last digits $(2 \sigma)$.

Accuracy and precision for $\mathrm{Pb}$ isotopes are better than $0.1 \%$ at the 2 sigma level, see appendix for analytical details. 
Table 2. Selected geochemical and isotopic analyses for dolerite dikes from the False Bay dike swarm

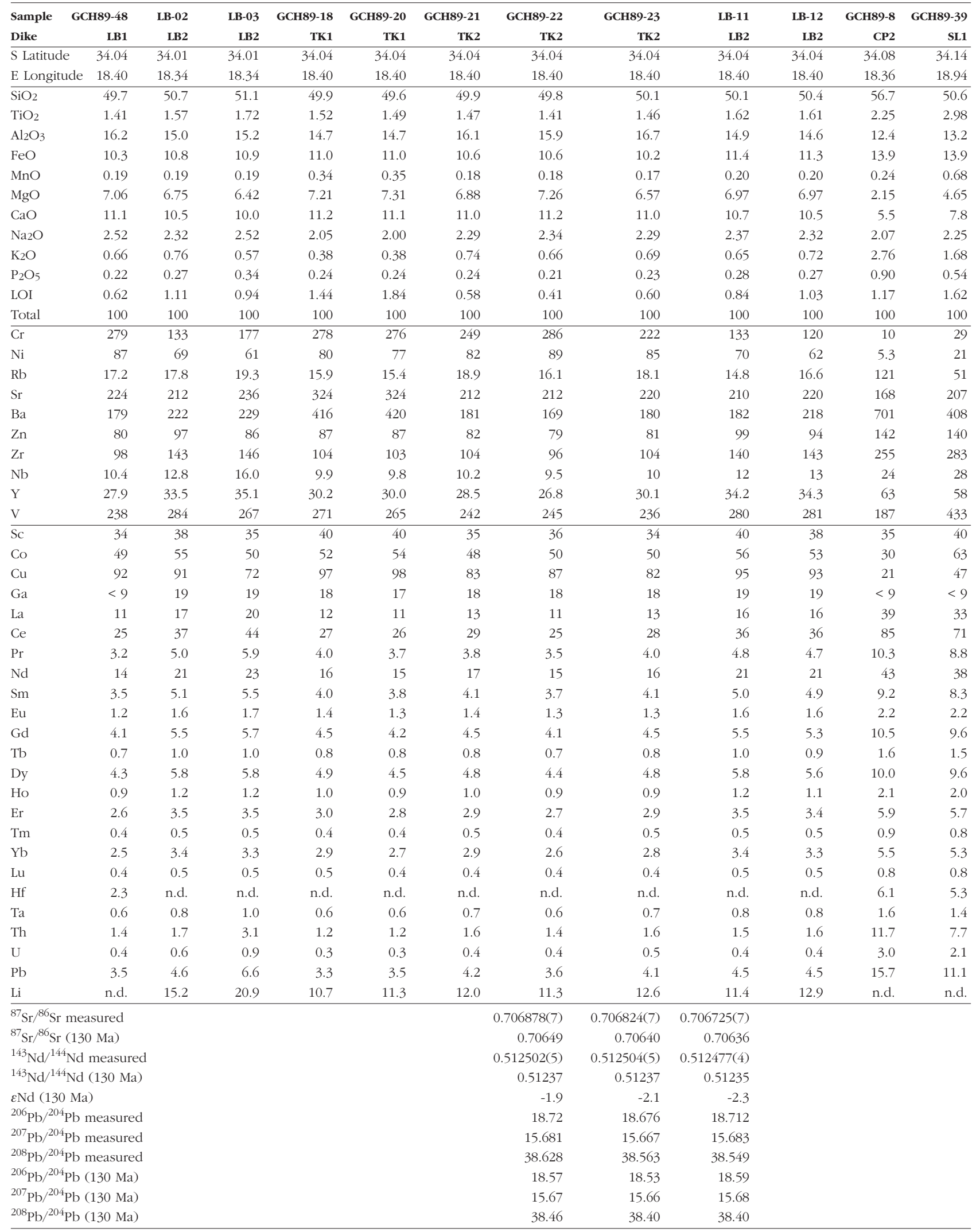

Sample locations in decimal degrees, based on the WG84 datum.

Major elements analysed by X-ray fluorescence, from Reid (1990), normalized to 100\% total and total Fe reported as FeO. n.d.= not determined. First block of trace elements (Cr to V) were analyzed by XRF (Reid, 1990); the second block by ICP-MS (ICP-OES for REE) at GFZ, see appendix. Measurement errors for $\mathrm{Sr}$ and $\mathrm{Nd}$ isotope ratios in parenthesis refer to last digits $(2 \sigma)$.

Accuracy and precision for $\mathrm{Pb}$ isotopes are better than $0.1 \%$ at the 2 sigma level, see appendix for analytical details. 
Table 3. Selected geochemical analyses for dolerite dikes from other localities south of the Orange River.

\begin{tabular}{|c|c|c|c|c|c|c|c|c|c|c|c|c|c|c|c|c|}
\hline \multirow[b]{2}{*}{ Sample } & \multicolumn{4}{|c|}{ Saldanha Bay } & \multicolumn{6}{|c|}{ Cedarberg Swarm } & \multicolumn{6}{|c|}{ Garies } \\
\hline & EG-001 & 1 EG-002 & EG-003 & EG-004 & GP-1 & GP-2 & GP-3 & GP-4 & GP-5 & GP-8 & CDB-1092 & CDB-1101 & CDB-1105 & CDB-1341 & I CDB-974 & CDB-978 \\
\hline Locality & & & & & Loc 3 & Loc 3 & Loc 4 & Loc 4 & Loc 4 & Loc 4 & Loc 1 & Loc 1 & Loc 1 & Loc 1 & Loc 2 & Loc2 \\
\hline S Latitude & 33.03 & 33.03 & 33.03 & 33.03 & 31.36 & 31.54 & 31.87 & 31.87 & 32.11 & 32.29 & 30.43 & 30.56 & 30.55 & 30.47 & 30.76 & 30.73 \\
\hline$\underline{\text { E Longitude }}$ & 17.96 & 17.96 & 17.96 & 17.96 & 18.60 & 18.96 & 18.79 & 18.79 & 19.38 & 19.66 & 17.46 & 17.41 & 17.41 & 17.37 & 17.90 & 17.86 \\
\hline $\mathrm{SiO}_{2}$ & 48.9 & 48.7 & 49.1 & 49.2 & 51.3 & 50.8 & 52.3 & 52.1 & 51.6 & 52.4 & 51.5 & 48.6 & 49.4 & 49.4 & 51.2 & 50.5 \\
\hline $\mathrm{TiO}_{2}$ & 3.56 & 3.62 & 3.59 & 3.56 & 1.07 & 1.63 & 1.86 & 1.77 & 1.97 & 1.99 & 1.30 & 1.12 & 1.28 & 1.15 & 1.19 & 1.14 \\
\hline $\mathrm{Al}_{2} \mathrm{O}_{3}$ & 12.6 & 12.8 & 12.8 & 12.7 & 13.8 & 13.3 & 12.6 & 12.8 & 12.7 & 12.5 & 13.4 & 13.3 & 13.2 & 13.3 & 13.7 & 13.7 \\
\hline $\mathrm{FeO}$ & 16.1 & 16.3 & 15.9 & 16.1 & 11.4 & 13.7 & 15.4 & 15.1 & 15.4 & 15.5 & 13.1 & 12.3 & 12.8 & 11.9 & 11.1 & 11.8 \\
\hline $\mathrm{MnO}$ & 0.24 & 0.25 & 0.24 & 0.24 & 0.19 & 0.21 & 0.22 & 0.21 & 0.21 & 0.22 & 0.20 & 0.21 & 0.21 & 0.20 & 0.19 & 0.20 \\
\hline $\mathrm{MgO}$ & 4.33 & 4.27 & 4.21 & 4.20 & 7.05 & 5.63 & 4.30 & 4.50 & 4.44 & 4.27 & 5.83 & 8.26 & 7.69 & 8.33 & 7.00 & 7.24 \\
\hline $\mathrm{CaO}$ & 9.27 & 9.23 & 9.26 & 9.14 & 11.05 & 9.92 & 8.44 & 8.46 & 8.49 & 8.35 & 9.58 & 11.27 & 11.47 & 11.81 & 11.14 & 10.63 \\
\hline $\mathrm{Na}_{2} \mathrm{O}$ & 2.30 & 2.23 & 2.26 & 2.17 & 2.49 & 2.82 & 2.87 & 3.20 & 3.10 & 2.93 & 3.14 & 2.46 & 2.18 & 2.18 & 2.56 & 2.87 \\
\hline $\mathrm{K}_{2} \mathrm{O}$ & 1.43 & 1.45 & 1.22 & 1.54 & 0.92 & 0.96 & 1.47 & 1.55 & 1.52 & 1.50 & 1.31 & 0.66 & 0.49 & 0.63 & 0.86 & 0.91 \\
\hline $\mathrm{P}_{2} \mathrm{O}_{5}$ & 0.64 & 0.66 & 0.65 & 0.65 & 0.16 & 0.29 & 0.30 & 0.27 & 0.33 & 0.33 & 0.20 & 0.11 & 0.13 & 0.11 & 0.17 & 0.15 \\
\hline LOI & 0.52 & 0.44 & 0.77 & 0.57 & 0.62 & 0.70 & 0.25 & 0.03 & 0.15 & 0.00 & 0.43 & 1.70 & 1.16 & 1.01 & 0.91 & 0.76 \\
\hline Total & 100 & 100 & 100 & 100 & 100 & 100 & 100 & 100 & 100 & 100 & 100 & 100 & 100 & 100 & 100 & 100 \\
\hline$\overline{\mathrm{Cr}}$ & 19 & 28 & 23 & 19 & 53 & 107 & 25 & 27 & 22 & 21 & 59 & 88 & 76 & 83 & 55 & 64 \\
\hline $\mathrm{Ni}$ & 32 & 34 & 36 & 32 & 82 & 77 & 57 & 59 & 56 & 55 & 72 & 72 & 67 & 72 & 85 & 80 \\
\hline $\mathrm{Rb}$ & 54 & 79 & 93 & 58 & 23 & 13 & 41 & 40 & 40 & 43 & 44 & 91 & 22 & 29 & 47 & 38 \\
\hline $\mathrm{Sr}$ & 226 & 202 & 238 & 218 & 180 & 166 & 157 & 148 & 152 & 142 & 172 & 98 & 101 & 103 & 264 & 210 \\
\hline $\mathrm{Ba}$ & 404 & 461 & 428 & 479 & n.d. & n.d. & n.d. & n.d. & n.d. & n.d. & 258 & 119 & 103 & 226 & 371 & 199 \\
\hline $\mathrm{Zn}$ & 159 & 158 & 169 & 152 & 77 & 102 & 91 & 83 & 86 & 96 & 71 & 91 & 81 & 65 & 87 & 69 \\
\hline $\mathrm{Zr}$ & 278 & 284 & 282 & 286 & 90 & 126 & 148 & 142 & 150 & 146 & 111 & 58 & 66 & 56 & 101 & 92 \\
\hline $\mathrm{Nb}$ & 24 & 24 & 24 & 24 & 7 & 9 & 12 & 12 & 12 & 12 & 9 & 4 & 5 & 4 & 7 & 6 \\
\hline Y & 61 & 63 & 62 & 63 & 20 & 32 & 38 & 37 & 40 & 40 & 29 & 21 & 24 & 21 & 22 & 20 \\
\hline $\mathrm{V}$ & 524 & 514 & 538 & 511 & 296 & 358 & 515 & 524 & 526 & 481 & 343 & 342 & 338 & 310 & 347 & 297 \\
\hline $\mathrm{Sc}$ & 39.3 & 37.7 & 41.5 & 36.4 & n.d. & n.d. & n.d. & n.d. & n.d. & n.d. & 40.8 & 44.5 & 45.6 & 43.9 & 41.4 & 40.2 \\
\hline Co & 49.5 & 53.3 & 53.6 & 50.4 & 47.9 & 46.3 & 42.3 & 44.9 & 45.4 & 48.6 & 48.4 & 52.3 & 49.7 & 50.1 & 48.4 & 45.5 \\
\hline $\mathrm{Cu}$ & 49.8 & 117 & 73.7 & 86.3 & 130 & 226 & 197 & 213 & 192 & 196 & 167 & 103 & 102 & 107 & 144 & 137 \\
\hline $\mathrm{Ga}$ & n.d. & n.d. & n.d. & n.d. & n.d. & n.d. & n.d. & n.d. & n.d. & n.d. & 19.6 & 17.6 & 17.5 & 16.9 & 19.1 & 18.6 \\
\hline $\mathrm{La}$ & 33.3 & n.d. & n.d. & n.d. & n.d. & n.d. & n.d. & n.d. & n.d. & n.d. & 17.0 & 6.4 & 7.3 & 6.4 & 15.1 & 12.9 \\
\hline $\mathrm{Ce}$ & 72.7 & n.d. & n.d. & n.d. & n.d. & n.d. & n.d. & n.d. & n.d. & n.d. & 38.6 & 15.6 & 17.8 & 15.7 & 34.6 & 30.0 \\
\hline $\operatorname{Pr}$ & 8.9 & n.d. & n.d. & n.d. & n.d. & n.d. & n.d. & n.d. & n.d. & n.d. & 4.8 & 2.1 & 2.4 & 2.1 & 4.3 & 3.7 \\
\hline $\mathrm{Nd}$ & 38.0 & n.d. & n.d. & n.d. & n.d. & n.d. & n.d. & n.d. & n.d. & n.d. & 19.8 & 9.4 & 10.7 & 9.3 & 17.5 & 15.3 \\
\hline $\mathrm{Sm}$ & 8.4 & n.d. & n.d. & n.d. & n.d. & n.d. & n.d. & n.d. & n.d. & n.d. & 4.8 & 2.8 & 3.2 & 2.7 & 4.2 & 3.8 \\
\hline $\mathrm{Eu}$ & 2.3 & n.d. & n.d. & n.d. & n.d. & n.d. & n.d. & n.d. & n.d. & n.d. & 1.4 & 0.9 & 1.0 & 1.0 & 1.3 & 1.2 \\
\hline Gd & 9.7 & n.d. & n.d. & n.d. & n.d. & n.d. & n.d. & n.d. & n.d. & n.d. & 5.6 & 3.6 & 4.1 & 3.6 & 4.5 & 4.1 \\
\hline $\mathrm{Tb}$ & 1.6 & n.d. & n.d. & n.d. & n.d. & n.d. & n.d. & n.d. & n.d. & n.d. & 0.9 & 0.6 & 0.7 & 0.6 & n.d. & n.d. \\
\hline Dy & 9.9 & n.d. & n.d. & n.d. & n.d. & n.d. & n.d. & n.d. & n.d. & n.d. & 5.6 & 4.0 & 4.5 & 4.0 & n.d. & n.d. \\
\hline Ho & 2.1 & n.d. & n.d. & n.d. & n.d. & n.d. & n.d. & n.d. & n.d. & n.d. & 1.2 & 0.8 & 0.9 & 0.8 & n.d. & n.d. \\
\hline $\mathrm{Er}$ & 6.2 & n.d. & n.d. & n.d. & n.d. & n.d. & n.d. & n.d. & n.d. & n.d. & 3.2 & 2.4 & 2.7 & 2.4 & n.d. & n.d. \\
\hline $\mathrm{Tm}$ & 0.9 & n.d. & n.d. & n.d. & n.d. & n.d. & n.d. & n.d. & n.d. & n.d. & 0.5 & 0.4 & 0.4 & 0.4 & n.d. & n.d. \\
\hline $\mathrm{Yb}$ & 5.9 & n.d. & n.d. & n.d. & n.d. & n.d. & n.d. & n.d. & n.d. & n.d. & 3.3 & 2.4 & 2.8 & 2.4 & 2.2 & 2.2 \\
\hline Lu & 0.9 & n.d. & n.d. & n.d. & n.d. & n.d. & n.d. & n.d. & n.d. & n.d. & 0.5 & 0.4 & 0.4 & 0.4 & 0.3 & 0.3 \\
\hline $\mathrm{Hf}$ & 6.5 & n.d. & n.d. & n.d. & n.d. & n.d. & n.d. & n.d. & n.d. & n.d. & n.d. & n.d. & n.d. & n.d. & n.d. & n.d. \\
\hline $\mathrm{Ta}$ & 1.4 & n.d. & n.d. & n.d. & n.d. & n.d. & n.d. & n.d. & n.d. & n.d. & 0.6 & 0.3 & 0.3 & 0.3 & 0.5 & 0.4 \\
\hline Th & 6.7 & 6.4 & 5.1 & 6.5 & $<3.5$ & $<3.5$ & $<3.5$ & $<3.5$ & 5.6 & $<3.5$ & 4.5 & 1.5 & 1.8 & 1.5 & 3.2 & 2.8 \\
\hline $\mathrm{U}$ & 1.6 & $<3.6$ & $<3.6$ & $<3.6$ & $<3.6$ & $<3.6$ & $<3.6$ & $<3.6$ & $<3.6$ & $<3.6$ & 1.1 & 0.4 & 0.5 & 0.4 & 0.8 & 0.6 \\
\hline $\mathrm{Pb}$ & 10.3 & 12.0 & 11.7 & 10.7 & $<2.2$ & $<2.2$ & 2.9 & 4.1 & 2.9 & $<2.2$ & 7.2 & 3.5 & 3.9 & 3.3 & 5.8 & 5. \\
\hline$\underline{\mathrm{Li}}$ & n.d. & n.d. & n.d. & n.d. & n.d. & n.d. & n.d. & n.d. & n.d. & n.d. & 15.0 & 45.9 & 12.6 & 8.2 & 9.8 & 12.8 \\
\hline
\end{tabular}

Sample locations in decimal degrees, based on the WG84 datum. Localities marked Loc 1, loc 3 etc. refer to Figure 4.

Major elements analysed by X-ray fluorescence at the University of Cape Town (UCT), normalized to $100 \%$ total, with total Fe reported as FeO. n.d. $=$ not determined.

First block of trace elements ( $\mathrm{Cr}$ to V) were analyzed by XRF at UCT, the second block by ICP-MS (ICP-OES for REE) at GFZ, see appendix.

in Table 3 for five localities. These include two en-echelon dike clusters striking northwest-southeast, called the Knersvlakte and Doring-Tanqua dikes on the Garies 1:250000 geologic map, (Figure 4, localities 3 and 4 , respectively); a group of small dikes from north of Koegel Fontein, referred to as the Garies dikes (localities 1 and 2 on Figure 4); and finally, a dike from the Saldanha Bay area which was thought to represent a member of the False Bay swarm on the grounds of its orientation and petrography (but contradicted by geochemical results see below).

\section{Geochemistry}

Earlier work on dike compositions from False Bay (Reid, 1990), the HOD (Trumbull et al., 2004b) and Mehlberg (Reid and Rex, 1994) was limited to XRF major element and trace element data. We selected samples from these studies for REE and additional trace element analyses and for $\mathrm{Sr}-\mathrm{Nd}-\mathrm{Pb}$ isotopic study. Our sample coverage in the HOD is extended by published data from dikes in the Huab-Brandberg area (Figure 2) by Thompson et al. (2001). We also present first geochemical results from dike localities in the Western Cape Province not 

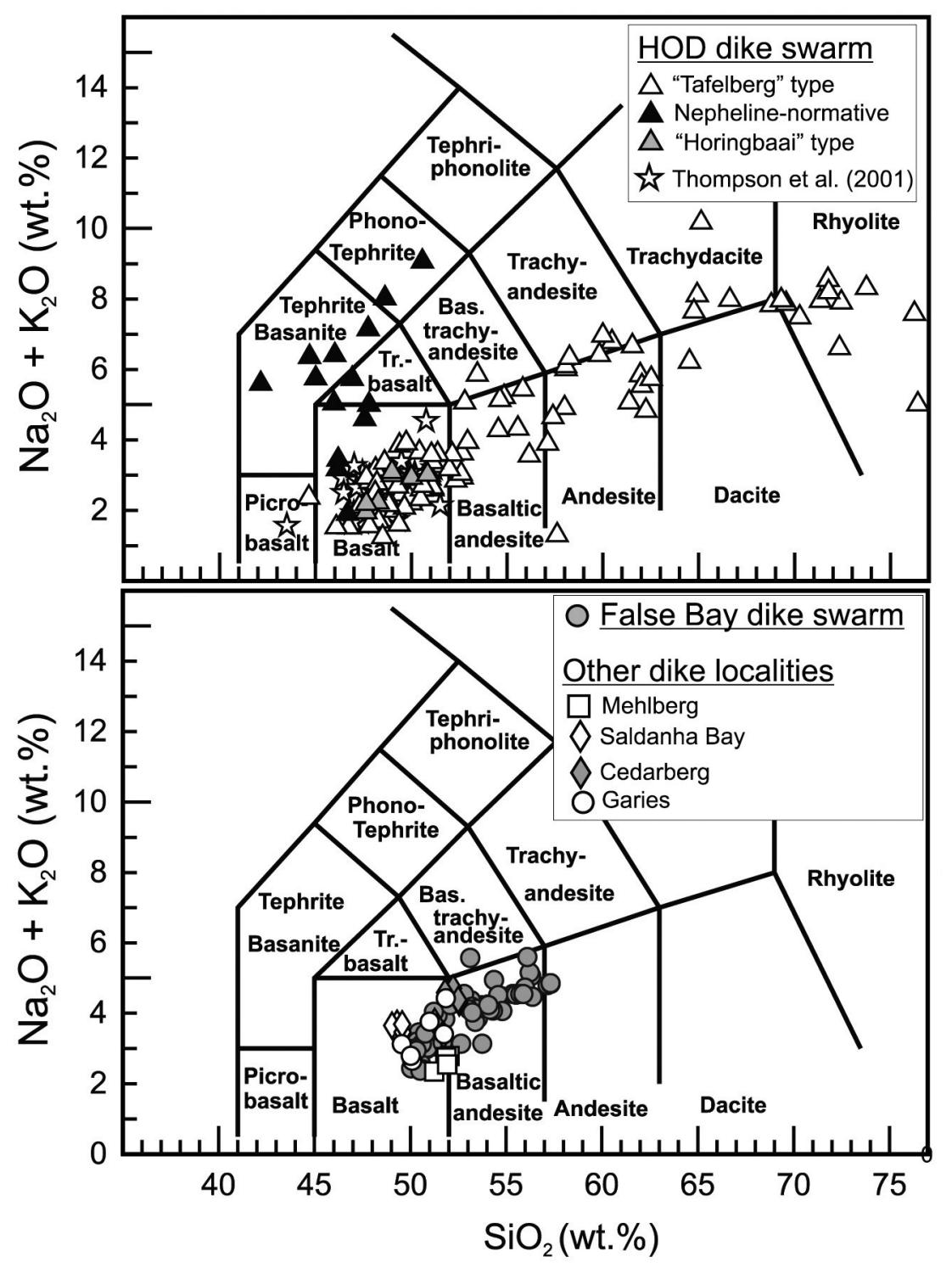

Figure 5. Whole-rock compositions of dikes from the HOD locality (top panel) and from localities south of the Orange River (bottom panel). The compositional variety of dikes from the HOD is much greater than in the other localities. The intermediate and silicic HOD dikes with $\mathrm{SiO}_{2}>57$ weight \% are not discussed in this paper (but see Trumbull et al., 2004b). White stars show composition of dolerite dikes reported from the HOD area by Thompson et al. (2001).

previously studied (Cedarberg, Garies, Saldanha Bay, see Figure 4). A description of analytical methods is given in the Appendix and representative analyses are presented in Tables 1, 2 and 3. In the geochemical plots and discussions that follow, all major element compositions have been recalculated to a 100 weight $\%$ volatile-free basis with total Fe reported as FeO.

\section{Whole-rock and olivine compositions}

The west coast dikes show an overwhelming dominance of subalkaline tholeiitic basalts and basaltic andesites in the total alkali-silica classification (Figure 5). The exceptional dikes with alkaline compositions (trachybasalts, basanite-tephrites) appear from present data to be confined to the HOD swarm. A further distinction of the HOD is the large number of intermediate and rhyolitic compositions (i.e., $\mathrm{SiO}_{2}>57$ weight \%). This paper is concerned exclusively with the dikes of basaltic and basaltic andesite compositions and their alkaline equivalents ( $<57$ weight $\% \mathrm{SiO}_{2}$ ), which for convenience will be referred to simply as dolerites. For a discussion of the intermediate and silicic dikes see Trumbull et al. (2004b).

The overall range of major element composition in the dolerite dikes is considerable (Figure 6) and most major and trace elements show good correlations with $\mathrm{MgO}$, consistent with igneous differentiation trends. Most samples in our data set have between 3 and 


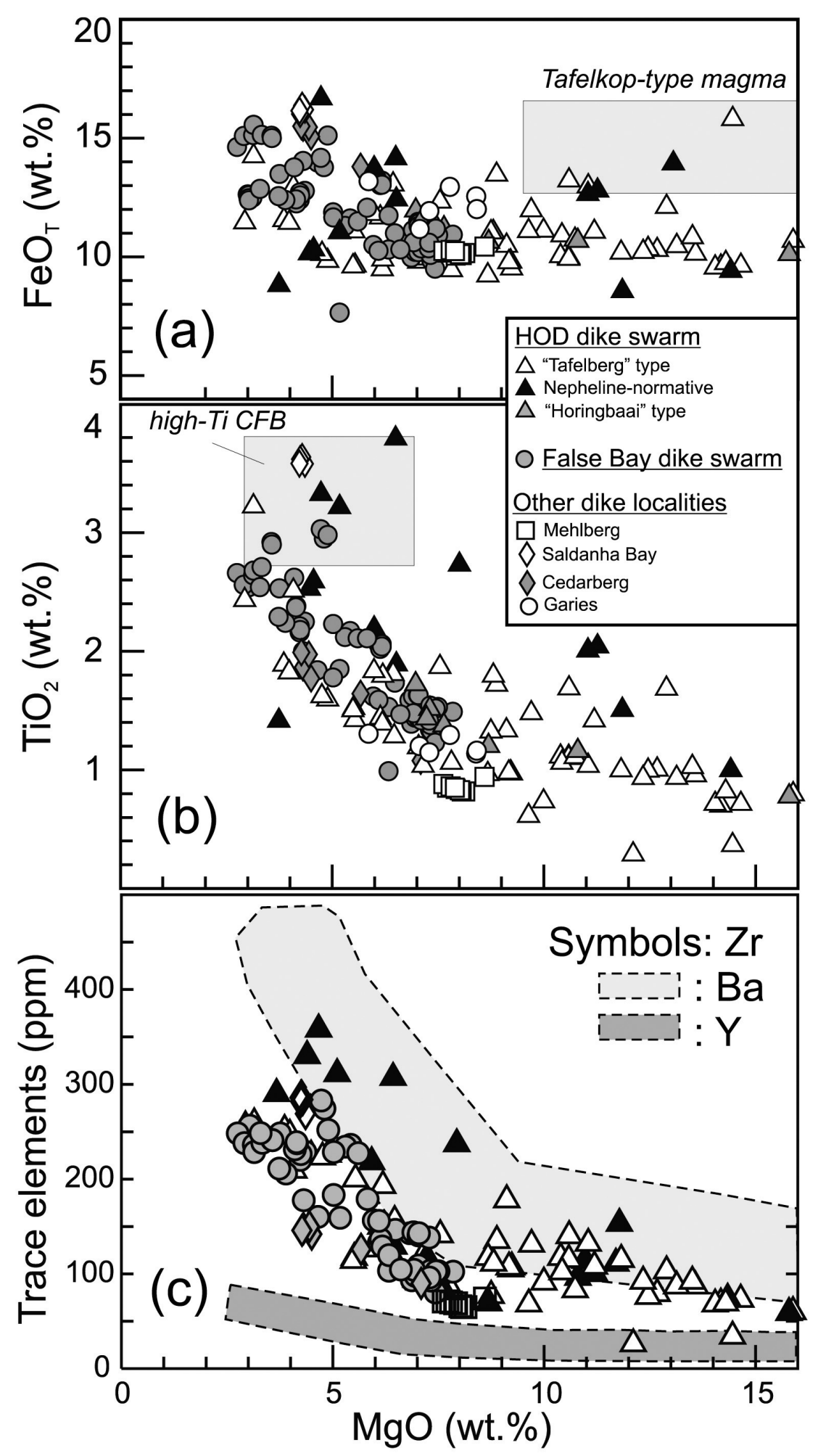

Figure 6. Selected whole-rock element variations of dolerite dikes against $\mathrm{MgO}$ as a differentiation index. Comparisons are shown in plot (a) with the Tafelkop or LTZ-H group of Etendeka basalts (data from Jerram et al., 1999 and Gibson, 2002); and in plot (b) with the highTi Etendeka basalts (data from Ewart et al., 2004). Note in plot (c) that three different elements are plotted. Symbols denote Zr concentration and shaded fields show variations for $\mathrm{Y}$ and Ba. The data from Thompson et al. (2001) plot within the range of HOD data from this study and are omitted for clarity. 


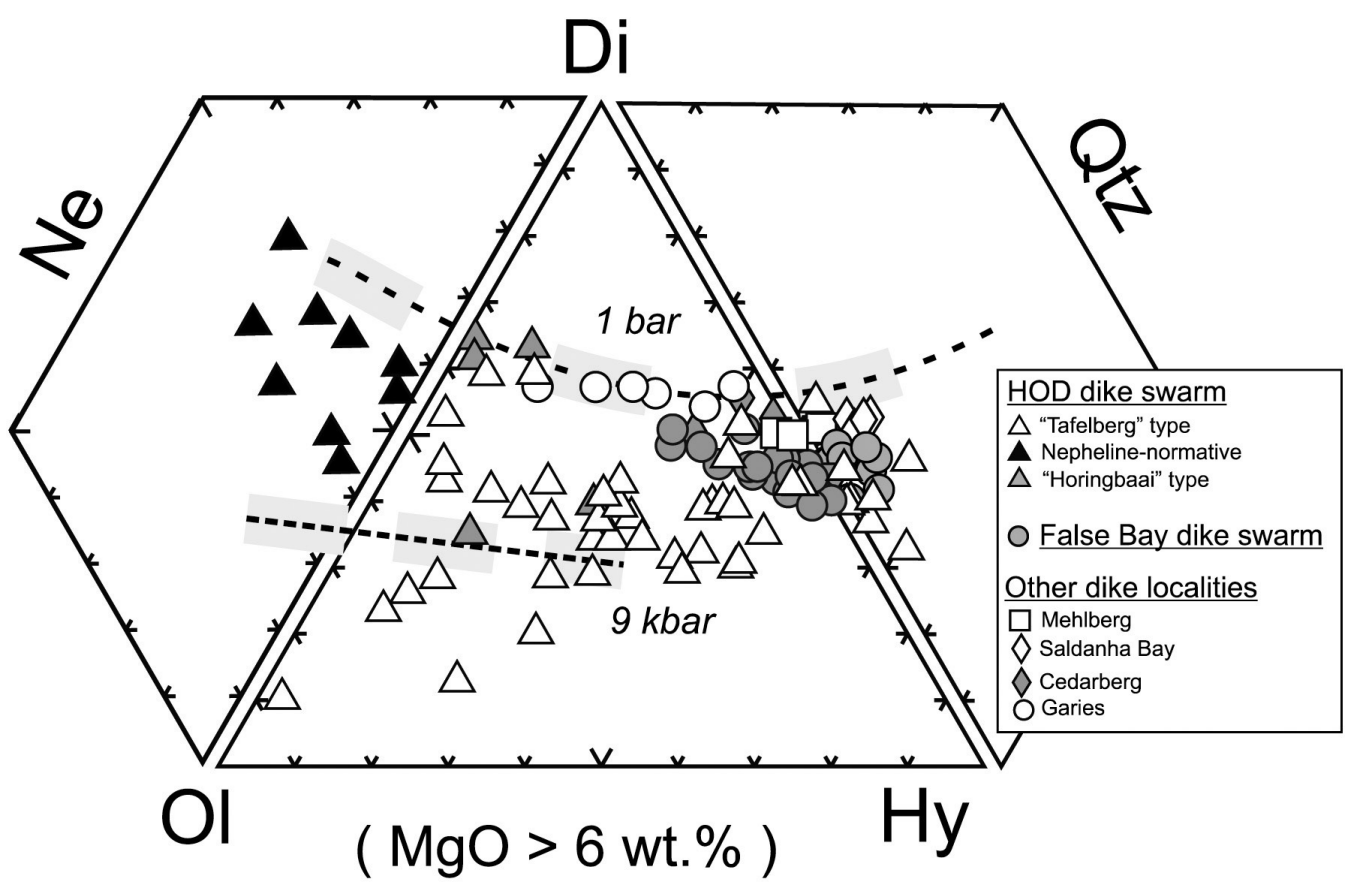

Figure 7. The CIPW normative compositions of west coast dolerite dikes, plotted for samples with > 6 weight $\%$ MgO (except Saldanha Bay at 4 weight \%). The norms assume a ratio of $\mathrm{Fe}_{2} \mathrm{O}_{3} / \mathrm{FeO}=0.15$. Dashed lines show compositional trends of experimental basaltic liquid cotectics at low and high pressure from Thompson et al. (1983; 2001). Most dolerites from False Bay and Garies localities and some Horingbaai-types from the HOD are consistent with low-pressure magma evolution before emplacement, whereas the HOD Tafelberg dikes plot closer to the 9kbar cotectic. Some samples deviate from cotectic compositions towards the olivine apex, indicating olivine accumulation.

16 weight $\% \mathrm{MgO}$, and a few exceptionally Mg-rich dikes contain up to 22 weight $\% \mathrm{MgO}$ due to olivine accumulation as discussed below. One of the most important observations of this study is the difference in the $\mathrm{MgO}$ contents of dikes from the northern and southern localities (Figure 6). None of the dikes sampled from south of the Orange River have more than 8 weight $\% \mathrm{MgO}$ and most are considerably less magnesian, whereas a large number of dolerites from the HOD exceed 10 weight \% MgO. Most of the Etendeka flood basalts (and Paraná basalts) have low to moderate $\mathrm{MgO}$ contents in the range of the False Bay swarm (Peate, 1997). The only known exception of high-Mg basalts are the olivine-bearing, so-called Tafelkop-type or LTZ-H basalts (Ewart et al., 1998; Jerram et al., 1999) which occur at the base of the Etendeka sequence in the Huab area (Figure 2). The Tafelkop basalts have distinctive isotopic and chemical compositions which led Gibson (2002) to propose that they represent direct melts of the rising Tristan plume head. However, the high-MgO dolerites from our study are not geochemically equivalent to the Tafelkop type. The dolerites lack the distinctive high FeO contents of Tafelkop lavas (see shaded field on Figure 6a), and their trace element and isotope compositions indicate closer affinities to the Horingbaai or Tafelberg magma types (see below). Most west coast dolerites in our compilation, except the Saldanha Bay dikes and HOD alkaline dikes, have low to moderate $\mathrm{TiO}_{2}$ concentrations (Figure 6b) and $\mathrm{Ti} / \mathrm{Zr}$ ratios (not shown), corresponding to the low-Ti magma series of the Paraná and Etendeka LIP (e.g., Peate, 1997; Marsh et al., 2001). For comparison, typical high-Ti type CFB compositions are illustrated by the shaded field on Figure 6b (Khumib lavas, Ewart et al., 2004).

As mentioned above, the systematic variations in major and trace element concentration with $\mathrm{MgO}$ are suggestive of gabbroic (plagioclase, pyroxene, olivine, oxide) fractionation trends, many of which show a distinctive change in slope at 6 to 8 weight $\% \mathrm{MgO}$ which is attributable to a change in the fractionating assemblage (Figures $6 \mathrm{~b}$ and $6 \mathrm{c}$, also $\mathrm{CaO}, \mathrm{Al}_{2} \mathrm{O}_{3}$ and others not shown). Details of magma evolution in the dikes are not at issue in this paper (see Reid, 1990 for a discussion of fractionation in the False Bay dike swarm). However, the compositional ranges need to be recognized and accounted for as a basis for regional comparisons. Since our interest is in the least differentiated compositions, it would be prudent to limit comparisons and interpretation to samples with $>8$ weight $\% \mathrm{MgO}$ but such samples are only present in the HOD swarm so we adopt a cutoff value of 6 weight $\% \mathrm{MgO}$ for much of the following discussion.

The CIPW normative compositions (Figure 7, samples $>6$ weight $\% \mathrm{MgO}$ ) underscore major-element differences between dolerites from the HOD swarm and 
those from False Bay and other localities from south of the Orange River. The latter cluster at the diopsidehypersthene join and near the composition of experimental cotectic melts at $1 \mathrm{~atm}$ pressure (dashed line, from Thompson et al., 1983; 2001). This suggests differentiation of these magmas at relatively low pressure within the crust, as concluded in previous studies of the low-Ti CFB magmas (e.g., Peate, 1997; Ewart et al., 1998). The HOD dolerites have a much greater range of normative composition, including several samples with up to $30 \mathrm{~mol} \%$ nepheline. Also, the olivine-normative HOD dolerites plot near the experimental 9 kbar cotectic line, suggesting a differentiation history at higher pressure. A final point about the CIPW compositions is that the whole-rock samples generally, excepting several HOD dikes with greater than 50\% normative olivine, are consistent with melt compositions. This is important because of the geodynamic significance of high-MgO magmas as discussed in a later section.

Olivine compositions from the HOD and False Bay localities are compared in Figure 8. The frequency histogram indicates that olivines with $85-90$ mol.\%
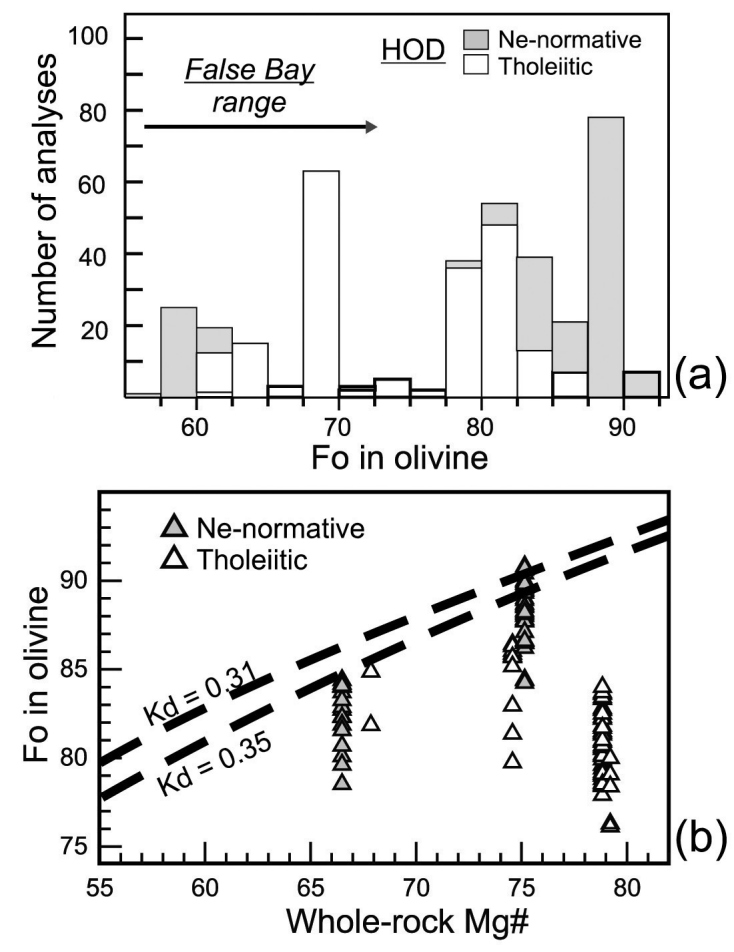

Figure 8. (a) Frequency histogram of olivine compositions (mole $\%$ forsterite) from the HOD tholeiitic and ne-normative dolerites (data from van Acken, 2006) compared with the range of olivine compositions reported for False Bay dikes by Reid (1990). (b) Plot of olivine forsterite contents for the most Mg-rich HOD samples against the whole-rock $\mathrm{Mg} \#$ (mole \% $\mathrm{MgO} / \mathrm{MgO}+\mathrm{FeO}$ assuming $\mathrm{Fe}_{2} \mathrm{O}_{3} / \mathrm{FeO}=0.15$ ). Dashed lines represent theoretical olivine-melt equilibrium compositions for $\mathrm{Kd}$ values of 0.31 and 0.35 . See text for discussion. forsterite are not uncommon in HOD dolerites, whereas olivines from the False Bay dikes are much less forsteritic (the arrow indicates compositional range from Reid, 1990). The inset to Figure 8 compares olivine and host-rock compositions for Mg-rich HOD rocks with reference to olivine-melt $\mathrm{Kd}$ lines for 0.31 and 0.35 for low and high pressure conditions, respectively (ca. 1 to 20 kbar, Ulmer, 1989). This plot confirms olivine accumulation for the two samples with the highest Mg-numbers, the host rock being richer in Mg than an equilibrium melt with those olivine compositions. Nevertheless, the key observation is that some samples with highly magnesian olivines (80-90\% forsterite) and Mg-numbers of 67 to 75 (12 to 15 weight \% MgO), are reasonably close to equilibrium compositions, thus confirming the presence of melt compositions in that range (see Thompson et al., 2001, who inferred parental Horingbaai-type melts with 15 weight \% MgO).

\section{Trace elements and magma types}

Primitive mantle (PM)-normalized trace element patterns of the west coast dolerite dikes indicate several distinct magma types (Figure 9), which is consistent with the radiogenic isotope variations described below. All samples plotted on Figure 9 have $>6$ weight $\% \mathrm{MgO}$ except Saldanha Bay where the cutoff had to be relaxed to 4 weight \% (see Figure 6). The most common trace element pattern overall, which is present all along the margin, is moderately inclined to the right (PM-normalized $\mathrm{La} / \mathrm{Yb}=3$ to 5 ) and shows prominent negative $\mathrm{Nb}$ and $\mathrm{Ta}$ anomalies and a positive $\mathrm{Pb}$ anomaly. This pattern is the most common one in the HOD swarm (Figure 9a) and it is the only type observed so far in the Garies dikes (Figure 9d) and in the Mehlberg and Saldanha localities (Figure 9f).

At least three magma types are distinguished among the HOD dolerites (Figures 9a to c). The most common of these is the "Tafelberg-type" (Figure 9a) whose trace element (and isotopic) characteristics resemble the main group of low-Ti Etendeka basalts, so-called "Tafelberg" type (e.g., Marsh et al., 2001), hence the name. Two other magma types among HOD dolerites have no known volcanic equivalents. One is the "Horingbaai type", named by Erlank et al. (1984) for compositionally distinctive (MORB-like) olivine-bearing dikes near Horingbaai which crosscut Etendeka lavas at that locality. The Horingbaai type was long assumed to be confined to the coastal dikes, but Thompson et al. (2001) found Horingbaai-type dikes more widely distributed inland and this is confirmed by our study. Compared with the Tafelberg dolerites, the Horingbaai type (Figure 9c) has relatively low PM-normalized element abundances and an overall flat distribution pattern (e.g., $\mathrm{La} / \mathrm{Yb}_{\mathrm{PM}}=2$ to 4 ) with the exception of positive $\mathrm{Pb}$ anomalies in many of the samples. Duncan et al. (1990) emphasized the "MORB-like" composition of Horingbaai dikes, and our data show that the Horingbaai-type dolerites from the HOD are similar to average E-MORB (Sun and McDonough, 1989) in terms 


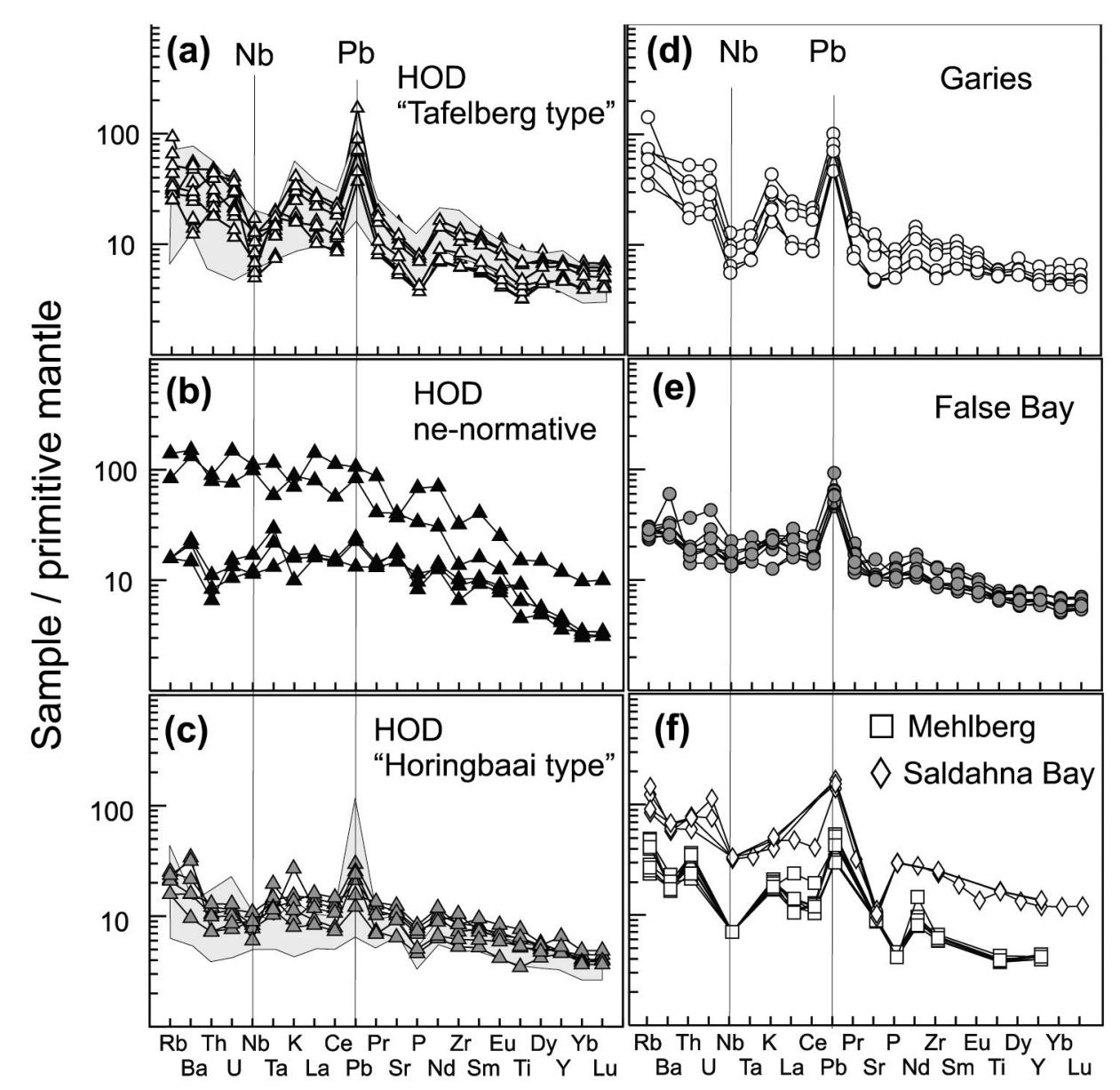

Figure 9. Plots of incompatible trace element compositions of west coast dikes ( $\mathrm{MgO}>6$ weight \%) normalized to primitive mantle (PM) values of Sun and McDonough (1989). The PM-normalized patterns discriminate distinct magma types within the HOD (compare Figure 11), and there is good agreement with our results and data from Thompson et al. (2001) shown in shaded fields for Horingbaai type (bottom left) and their Tafelberg + Nil Desperandum magma types (top left). Note the similarity between PM-normalized patterns of the HOD Tafelberg dikes and dolerites from Garies, Mehlberg and Saldanha Bay localities. False Bay dolerites differ from the Tafelberg type in the lack of a negative Nb-Ta anomaly.

of the trace element patterns, apart from the positive $\mathrm{Pb}$ anomalies. The third HOD magma type comprises the alkaline compositions and is termed the nephelinenormative type based on the CIPW norm (Figure 7). Trace element patterns of nepheline-normative dolerites form two separate groupings on Figure 9b, both with relatively smooth PM-normalized patterns, but with a 10fold difference in abundances. The low-abundance alkaline group resembles the Horingbaai-type in trace element pattern $\left(\mathrm{La} / \mathrm{Yb}_{\mathrm{PM}}=5\right)$, whereas the other group has much steeper patterns $\left(\mathrm{La} / \mathrm{Yb}_{\mathrm{PM}}=15\right.$ to 24$)$ which resemble those of the lamprophyres, tephrites and alkaline gabbros from Damaraland intrusive complexes (Trumbull et al., 2003). For completeness we note that Thompson et al. (2001) proposed a further magma type for dikes from an area NE of Huab (Figure 2), which they termed "Nil Desperandum". This magma type is currently represented by only a few samples and needs better definition but the present data show that Nil Desperandum dolerites have similar but slightly flatter
PM-normalized patterns as the Tafelberg type (lower Pb, $\mathrm{Rb}, \mathrm{U}, \mathrm{Th}$ ), and less radiogenic $\mathrm{Sr}$ isotope ratios (see below). None of the dikes in our study correspond to the "Nil Desperandum" type as defined in Thompson et al. (2001).

The trace element patterns of False Bay dikes (Figure 9e) are very similar to one another and they vary only in abundance, suggesting the presence of a single magma type (see Reid, 1990). The False Bay trace element characteristics resemble those of Tafelberg-type magmas in several ways $\left(\mathrm{La} / \mathrm{Yb}_{\mathrm{PM}}=5\right.$, strong positive $\mathrm{Pb}$ anomaly) but unlike the Tafelberg type, there is no negative $\mathrm{Nb}$ and $\mathrm{Ta}$ anomaly developed in the False Bay patterns.

\section{Radiogenic isotopes}

Initial $\mathrm{Sr}$ and $\mathrm{Nd}$ isotope data from representative samples of the HOD (Table 1) and False Bay dolerites (Table 3) are shown in Figure 10, with the corresponding trace element patterns illustrated in 


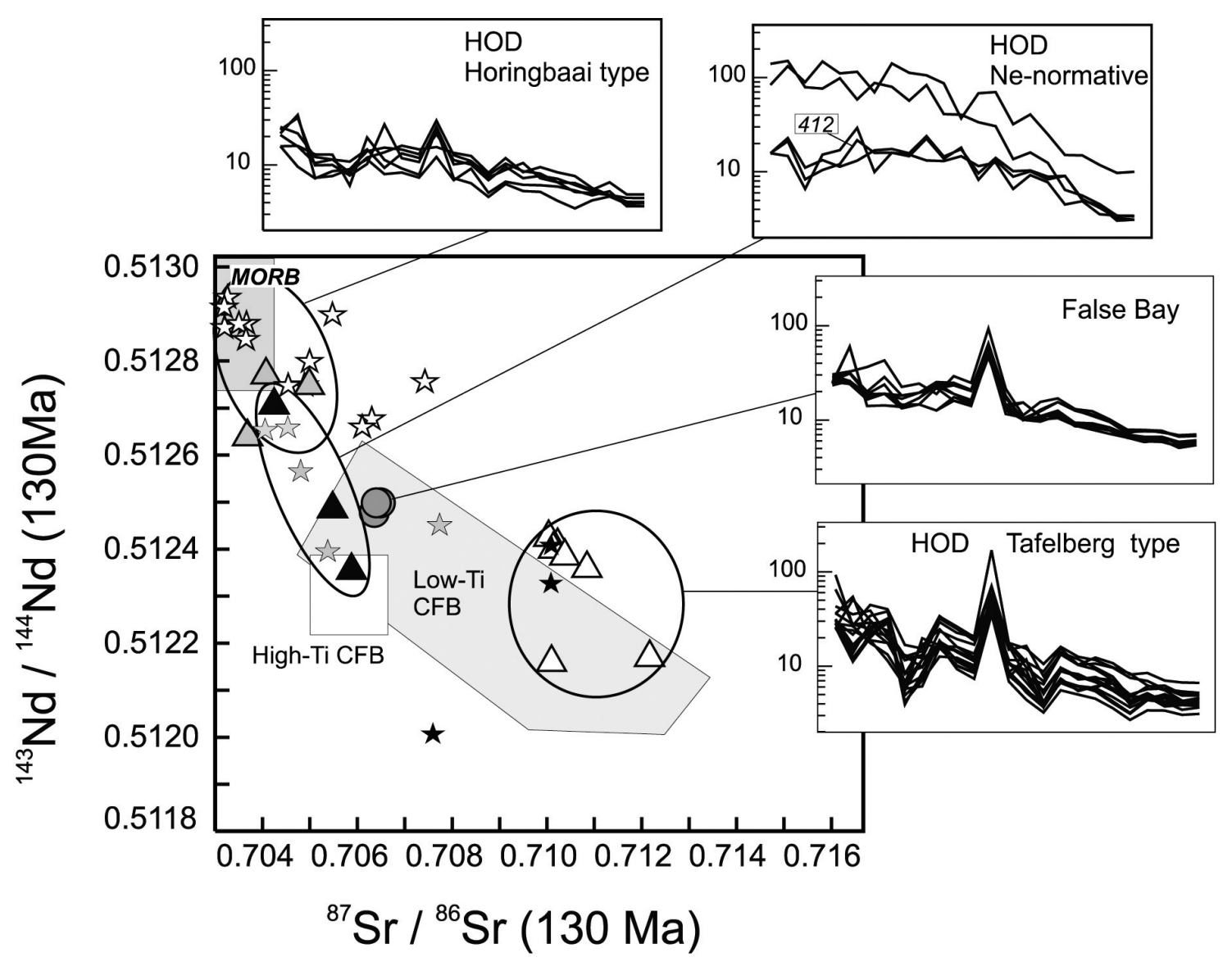

Figure 10. The whole-rock ${ }^{87} \mathrm{Sr} /{ }^{86} \mathrm{Sr}$ and ${ }^{143} \mathrm{Nd} /{ }^{144} \mathrm{Nd}$ ratios (age-corrected for $130 \mathrm{Ma}$ ) of dikes from the HOD swarm and from False Bay are plotted along with compositional fields for the main flood basalt types (high-Ti and low-Ti CFB) from the Etendeka province and MORB (Etendeka data from Ewart et al., 1998; 2004 and MORB from Hoffmann, 1997). Data from Thompson et al. (2001) are shown in stars for Horingbaai (white) Tafelberg (black) and Nil Desperandum (gray) magma types. The isotopic compositions correlate with geochemical magma types discriminated by trace element patterns, as shown by PM-normalized multielement diagrams (from Figure 9).

the insets. Again, all samples plotted have $\mathrm{MgO}>6$ weight $\%$. Distinctive for the Horingbaai-type dikes from the HOD (gray triangles: our data; white stars: Thompson et al., 2001) are the highly radiogenic Nd and non-radiogenic $\mathrm{Sr}$ isotope ratios, which overlap on Figure 10 with the MORB field and confirm a MORBsource affinity emphasized by Duncan et al. (1990). At the other extreme in Sr-Nd isotope composition are the Tafelberg-type HOD dolerites (white triangles: our data, black stars: Thompson et al., 2001), whose high radiogenic $\mathrm{Sr}$ and low radiogenic $\mathrm{Nd}$ ratios are similar to the most enriched (radiogenic Sr, non-radiogenic Nd) low-Ti CFB lavas (shaded field on Figure 10). The alkaline, ne-normative HOD dikes (black triangles) and the "Nil Desperandum" types (gray stars, Thompson et al., 2001) plot between the range of Horingbaai-type dolerites and the compositional field for high-Ti basalts (data from Ewart et al., 2004). Finally, the False Bay dolerites are isotopically distinct from both the Tafelberg and Horingbaai-types. They form a tight cluster in the SrNd diagram at the depleted end of the low-Ti CFB field.

The $\mathrm{Pb}$ isotope compositions from the HOD dikes (Figure 11) cover a wide range, extending both to more radiogenic and less radiogenic compositions than the low-Ti Etendeka CFB field. The Tafelberg-type dolerites (white triangles, black stars) have the most radiogenic $\mathrm{Pb}$ isotope compositions, plotting beyond the CFB field and into the range of the Damara basement rocks (metasediments and granites, see caption). The Horingbaai-type HOD dikes (gray triangles, white stars), which defined a distinctive cluster in the $\mathrm{Nd}$ and $\mathrm{Sr}$ isotope plot (Figure 10), vary much more in $\mathrm{Pb}$ isotope compositions and show only marginal correspondence with the MORB field in ${ }^{207} \mathrm{~Pb} /{ }^{204} \mathrm{~Pb}$ and ${ }^{208} \mathrm{~Pb} /{ }^{204} \mathrm{~Pb}$. In fact, some Horingbaai samples are nearly as rich in radiogenic $\mathrm{Pb}$ as the Tafelberg type and overlap with the basement field. The ne-normative and "Nil Desperandum" dolerites from the HOD (black 


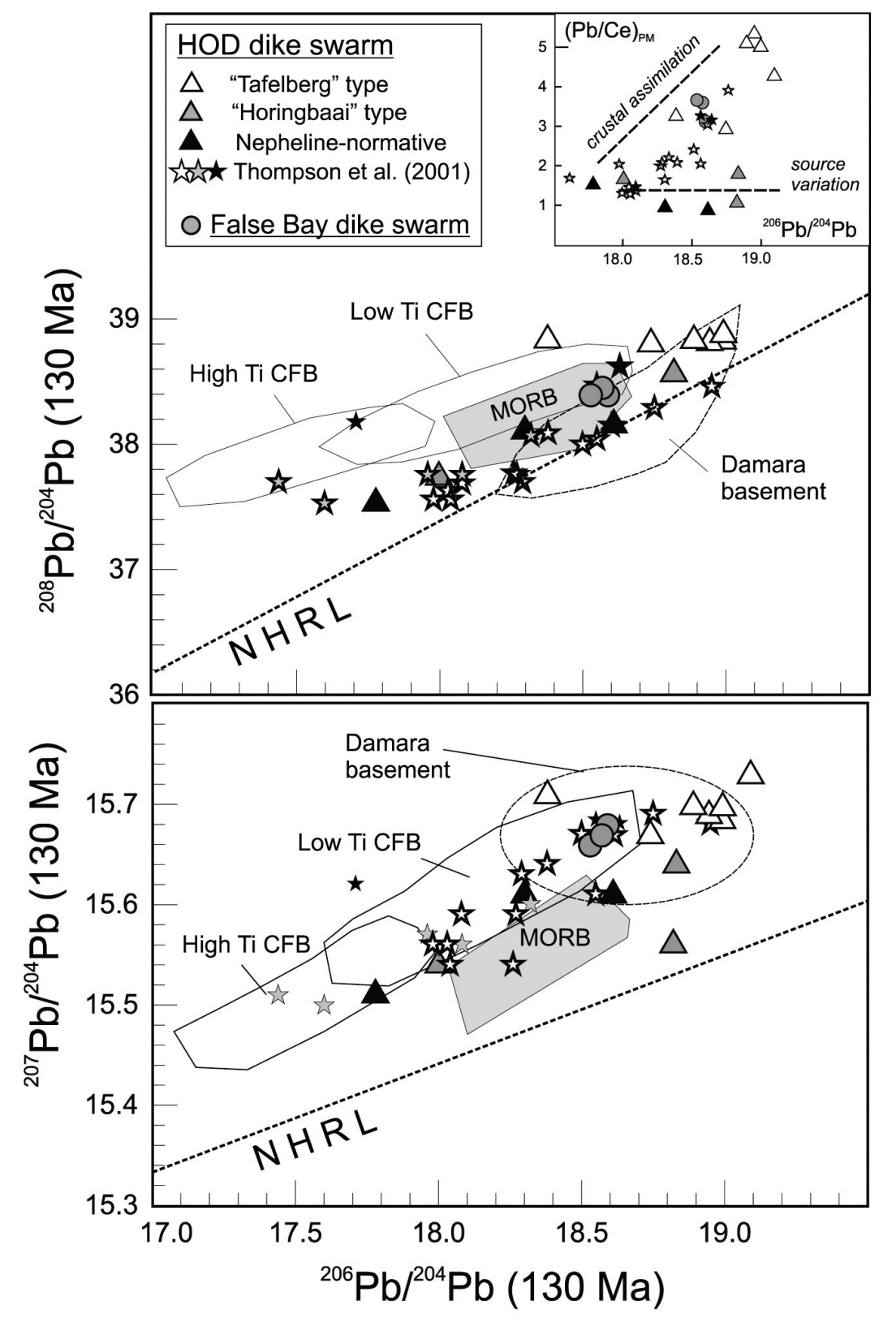

Figure 11. The whole-rock $\mathrm{Pb}$ isotope ratios (calculated for $130 \mathrm{Ma}$ ) of dikes from the HOD and False Bay swarms, with data from Thompson et al. (2001) in stars as in Figure 10. Data sources for high-Ti and low-Ti CFB and MORB are the same as Figure 9. The compositional field for Damara Basement includes metasedimentary rocks and S-type granites (from McDermott et al., 1996 and Jung et al., 2000, respectively). The Northern Hemisphere Reference Line (NHRL) is from Hart (1988). Several samples of both Tafelberg and Horingbaai types plot in the field of Damara basement, suggesting their radiogenic $\mathrm{Pb}$ isotope compositions result from crustal assimilation. The inset shows the good correlation of high ${ }^{206} \mathrm{~Pb} /{ }^{204} \mathrm{~Pb}$ ratios with $\mathrm{PM}$-normalized $\mathrm{Pb} / \mathrm{Ce}$ ratios, a measure of the positive $\mathrm{Pb}$ anomalies (see Figure 9).

triangles, gray stars) also vary widely in $\mathrm{Pb}$ isotope ratios. Most samples overlap with the other magma types and with the low-Ti CFB, but a few have less radiogenic $\mathrm{Pb}$ isotope ratios and extend into the compositional field of high-Ti flood basalts. Finally, the False Bay dolerites have uniform $\mathrm{Pb}$ isotope compositions, which plot at the radiogenic end of the low-Ti CFB field in Figure 11 and also within the Damara basement field. Recall that the False Bay dikes plot at the opposite, depleted, end of the low-Ti CFB field in terms of their $\mathrm{Sr}$ and $\mathrm{Nd}$ isotope ratios (Figure 10).

In summary, the different magma types from the HOD and False Bay dikes have much less distinctive isotopic compositions of $\mathrm{Pb}$ compared with $\mathrm{Sr}$ and $\mathrm{Nd}$. Furthermore, all dolerite groups correspond at least partly with the more radiogenic CFB compositions and Damara basement rocks in terms of $\mathrm{Pb}$ isotopes, whereas the same samples plot at the depleted end of 


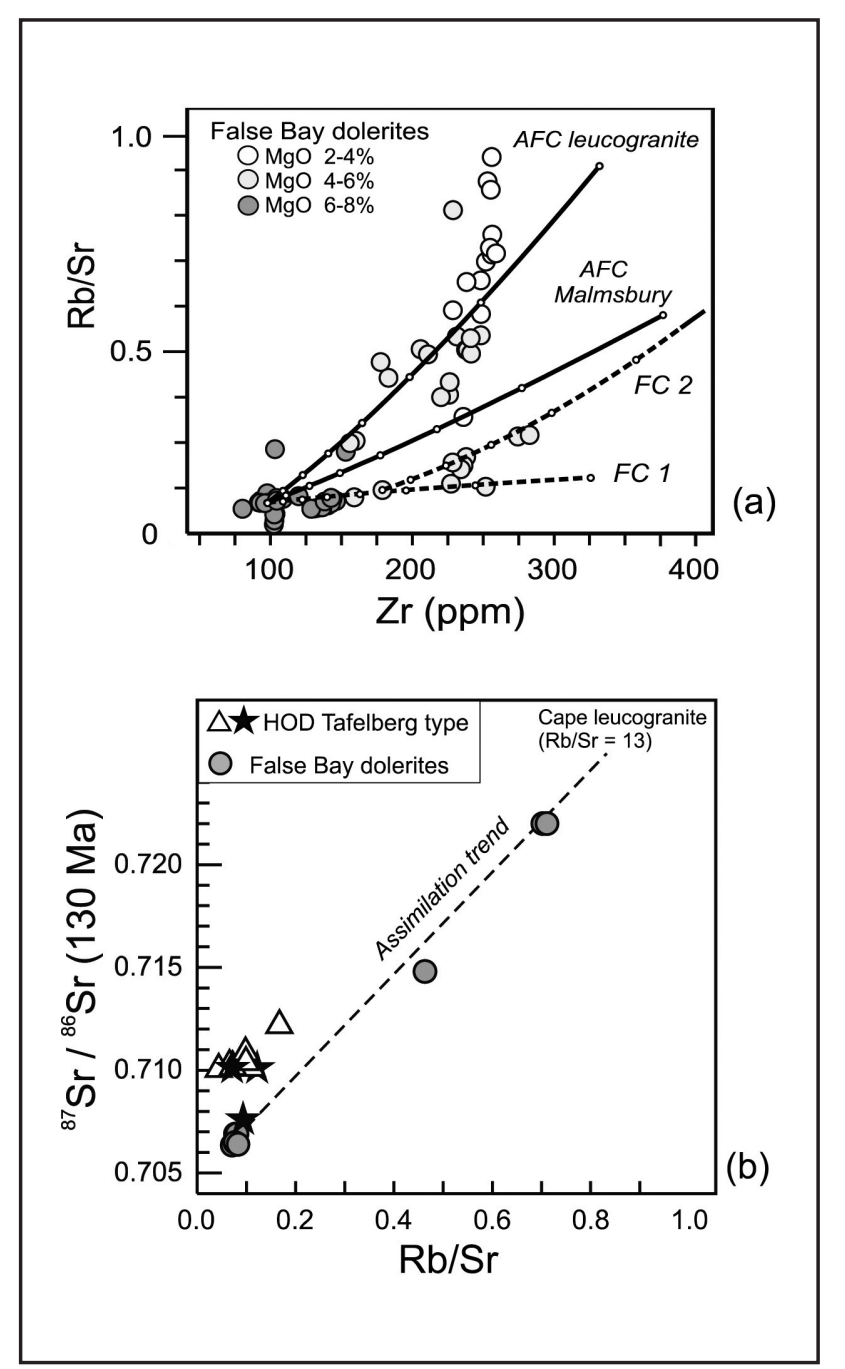

Figure 12. Trace element and Sr- isotopic evidence for crustal assimilation in the False Bay dikes. (a) curve FC 1 illustrates early cpx-ol dominated crystallization (bulk distribution coefficients $\mathrm{D}_{\mathrm{Sr}}=0.5, \mathrm{D}_{\mathrm{Zr}}=0$ and $\mathrm{D}_{\mathrm{Rb}}=0$ ), and $\mathrm{FC} 2$ assumes crystallization of only plagioclase $\left(D_{\mathrm{Sr}}=2, \mathrm{D}_{\mathrm{Zr}}=0\right.$ and $\left.\mathrm{D}_{\mathrm{Rb}}=0\right)$. The curves extend from $\mathrm{f}=0$ (melt) to $\mathrm{f}=0.3$ ( $70 \%$ crystallized) with tics every $10 \%$. The combined assimilation and fractional crystallization (AFC) curves are calculated for Cape leucogranite (Allsopp and Kolbe, 1965) and Malmesbury metasediments (Kolbe and Taylor, 1966) for a simple assumption of one-stage evolution $\left(\mathrm{D}_{\mathrm{Sr}}=1.2, \mathrm{D}_{\mathrm{Zr}}=0\right.$ and $\mathrm{D}_{\mathrm{Rb}}=0$ ), and for a value of 0.1 for $\mathrm{r}$ (mass assimilated/mass crystallized). AFC curves also extend from $\mathrm{f}=0$ to $\mathrm{f}=0.3$. (b) the near-linear correlation between $\mathrm{Rb} / \mathrm{Sr}$ and initial ${ }^{87} \mathrm{Sr} /{ }^{86} \mathrm{Sr}$ ratios (includes 3 unpublished analyses of low-Mg samples from University of Cape Town) support the assimilation model for False Bay magmas and Cape leucogranite. A correlation is also observed for the Tafelberg-type HOD dikes (triangles: this study, stars: Thompson et al., 2001) but the range is much smaller.

the CFB array and some overlap with MORB in terms of Sr-Nd isotopes. This contrasting behavior is evidence for assimilation of crustal $\mathrm{Pb}$, as supported by the plot of initial ${ }^{206} \mathrm{~Pb} /{ }^{204} \mathrm{~Pb}$ ratio against $(\mathrm{Pb} / \mathrm{Ce})_{\mathrm{PM}}$ (inset Figure 11). The $(\mathrm{Pb} / \mathrm{Ce})_{\mathrm{PM}}$ ratio is proportional to the size of the positive $\mathrm{Pb}$ anomaly on $\mathrm{PM}$-normalized trace element patterns (Figure 9) and we observe a good positive correlation with ${ }^{206} \mathrm{~Pb} /{ }^{204} \mathrm{~Pb}$ ratios (and with ${ }^{87} \mathrm{Sr} /{ }^{86} \mathrm{Sr}$ and ${ }^{143} \mathrm{Nd} /{ }^{144} \mathrm{Nd}$, not shown) except for samples with $(\mathrm{Pb} / \mathrm{Ce})_{\mathrm{PM}}$ near unity (i.e. those lacking positive $\mathrm{Pb}$ anomalies). Interestingly, the latter (ne-normative dolerites and some Horingbaai types) still show a wide a range in ${ }^{206} \mathrm{~Pb} /{ }^{204} \mathrm{~Pb}$ ratios, which is likely to be source related.

\section{Discussion: magma compositions at high-flux and low-flux margin segments}

The dominant composition of Early Cretaceous dolerite dikes along the Atlantic margin of Namibia and South Africa is low-Ti tholeiite. In terms of trace element and $\mathrm{Sr}-\mathrm{Nd}-\mathrm{Pb}$ isotopic compositions, this magma type is similar to the main type of low-Ti CFB magma from the Etendeka province, and it is worth pointing out that essentially the same magma type dominates in the Paraná CFB (Gramado type: Peate, 1997) and in the Karoo basalts and sills as well ("Lesotho type": Marsh et al., 1997). The dolerite dikes from the northern and southern segments of the Atlantic margin have many compositional features in common, but in this discussion we emphasize two fundamental differences. One is the contrasting diversity of magma types present and the other is the unequal distribution of high-Mg (picritic) magmas.

\section{Compositional diversity and mantle vs. crustal input}

The HOD dolerites comprise at least three magma types based on their CIPW norm compositions, trace element patterns and radiogenic isotope ratios (Figures 6, 7, 9 and 10) whereas the False Bay swarm and other localities in the southern margin apparently contain only one. As described before, the tholeiitic, low-Ti, Tafelberg type dikes are compositionally very similar to the Etendeka basalts of that name, which led to the suggestion that the Tafelberg dikes are feeders for noweroded basalt fields (Erlank et al., 1984; Marsh et al., 2001, Trumbull et al., 2004b). The Horingbaai and nepheline-normative types have no known equivalents among the CFB units and there is some geologic evidence that the two may post-date the main tholeiitic sequence. First, Horingbaai dikes at the type locality cut Etendeka lavas (Erlank et al., 1984); and second, several of the Damaraland complexes display a clear intrusive sequence of early tholeiitic magma and later mafic alkaline units (Messum: Harris et al., 1999; Okenyenya: Milner and le Roex, 1996; Erongo: Wigand et al., 2004).

The diversity of compositions in the HOD swarm is perhaps not surprising considering the variety of magmas recognized among contemporary Etendeka basalts and mafic intrusive complexes (e.g., Marsh et al., 2001; Trumbull et al., 2003). Despite many studies of magma genesis in the Etendeka Province (e.g., Milner and le Roex, 1996; Ewart et al., 1998; 2004; Harris et al., 1999; Thompson et al., 2001; Trumbull et al., 2003) there is no agreement on how much compositional diversity is related to different mantle components and how much 


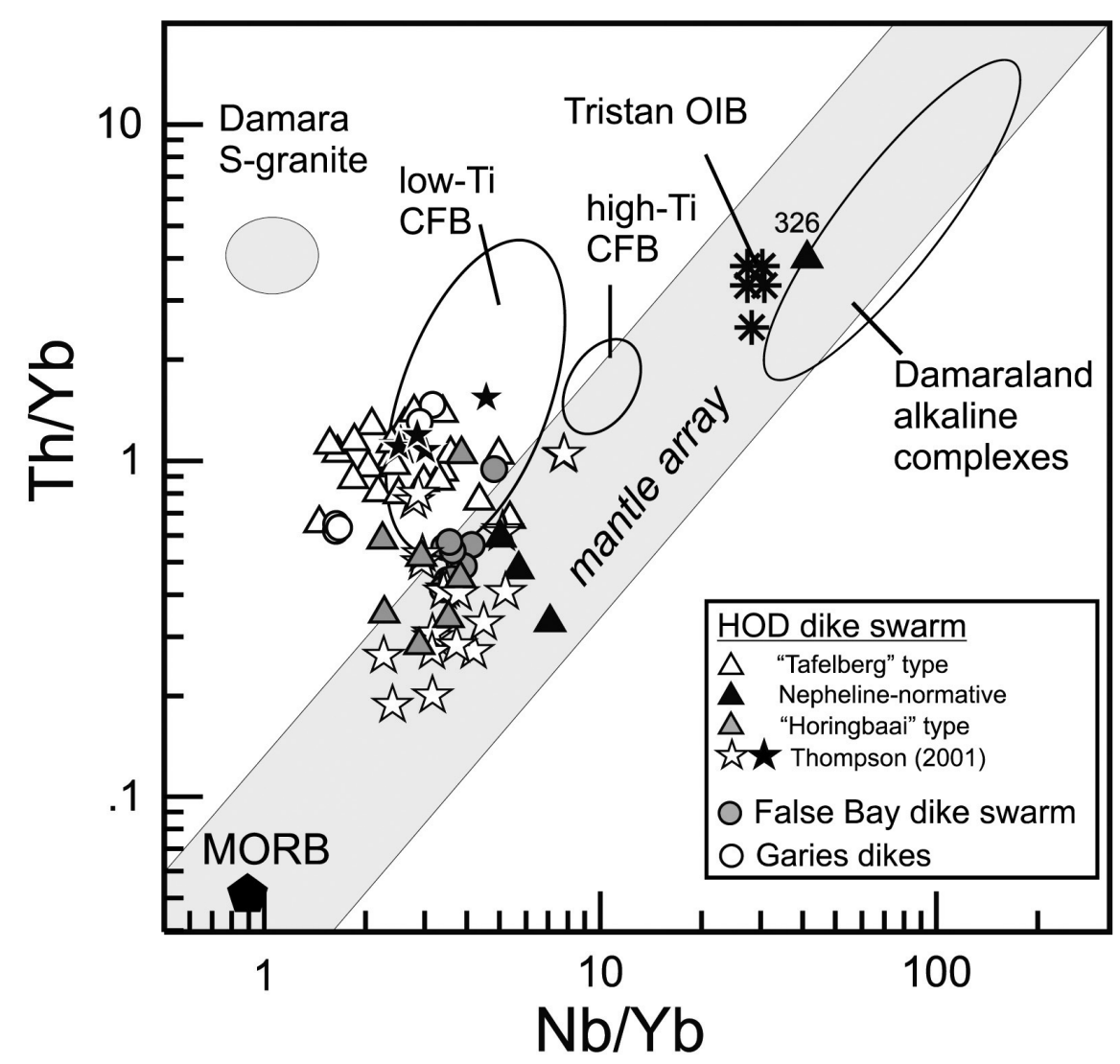

Figure 13. Summary diagram showing incompatible trace element ratios for the least-contaminated dolerite dikes $(\mathrm{MgO}>6 \mathrm{weight} \%$, Rb/Sr $<0.2$ ). The gray field labelled mantle array represents compositions of the sub- lithospheric mantle defined by MORB, Tristan island OIB (Hoffmann, 1997), and plume-related alkaline complexes from the Damaraland of Namibia (Harris et al., 1999; Trumbull et al., 2003). Fields for low-Ti and high-Ti lavas from the Etendeka province are from Ewart et al. (2004), and the composition of Damara S-type granites from Jung et al. (2000). The False Bay dolerites as well as the ne-normative and Horingbaai-type HOD dolerites plot in the mantle array. However, Garies dolerites and Tafelberg-type HOD dolerites have higher Th/Yb and lower Nb/Yb ratios, like the low-Ti CFB (see text). Open and filled stars are Horingbaai-type and Tafelberg type type dolerites, respectively, from Thompson et al. (2001).

is imposed by interaction with the continental crust (see Thompson et al. 2007). Our study of dolerite dikes in the HOD and False Bay swarms was not designed to derive genetic models for the various magma types, but the results can contribute some new arguments and observations relevant to the crust vs. mantle debate. The effects of crustal assimilation on dolerites in the False Bay and HOD localities are readily apparent if the compositional restriction of $>6$ weight $\% \mathrm{MgO}$ is relaxed. Figure 12a illustrates variations in $\mathrm{Rb} / \mathrm{Sr}$ and $\mathrm{Zr}$ in the False Bay dolerites ( $\mathrm{MgO}$ from 2 to 8 weight \%) and curves calculated for two-stage fractional crystallization (FC 1 and FC 2) and for fractional crystallization combined with assimilation (AFC) of local basement rocks (Malmsbury metasediments and Cape leucogranite, see caption). The samples with $\mathrm{MgO}>6$ weight $\%$ (dark shading) define a trend consistent with fractionation of mainly $\mathrm{Fe}-\mathrm{Mg}$ silicates and minor plagioclase (curve FC 1, bulk distribution coefficients $\mathrm{D}_{\mathrm{Rb}}=0, \mathrm{D}_{\mathrm{Zr}}=0, \mathrm{D}_{\mathrm{Sr}}=0.5$ ). The crystallizing assemblage will of course change during fractionation (see kinked trends on Figure 6) and curve FC 2 illustrates an extreme scenario assuming that intermediate melts fractionate only plagioclase $\left(D_{R b}=0, D_{Z r}=0, D_{S r}=2\right)$. This model is shown to demonstrate the maximum increase in $\mathrm{Rb} / \mathrm{Sr}$ ratios attainable by fractional crystallization, which falls well short of the data trend. Assimilation of a Rb-rich component is required and the AFC curves show that assimilation of evolved upper-crustal granite (Cape leucogranite) is a feasible scenario (see caption for details). Unequivocal evidence for crustal assimilation is the near-linear correlation of ${ }^{87} \mathrm{Sr} /{ }^{86} \mathrm{Sr}$ ratios and the $\mathrm{Rb} / \mathrm{Sr}$ ratio in the False Bay dolerites (Figure 12b), which fits a two-component mixing curve between the leastevolved samples and the Cape leucogranite. Tafelbergtype dolerites from the HOD also show a good correlation of $\mathrm{Sr}$ isotope ratios and $\mathrm{Rb} / \mathrm{Sr}$ but the total variation is small because only samples with $>7$ weight $\% \mathrm{MgO}$ were chosen for isotopic analysis. The main point of Figure 12 is that effects of crustal assimilation can be recognized and effectively removed by restricting samples to $\mathrm{MgO}>6$ weight $\%$ and $\mathrm{Rb} / \mathrm{Sr}<0.1$. When this 


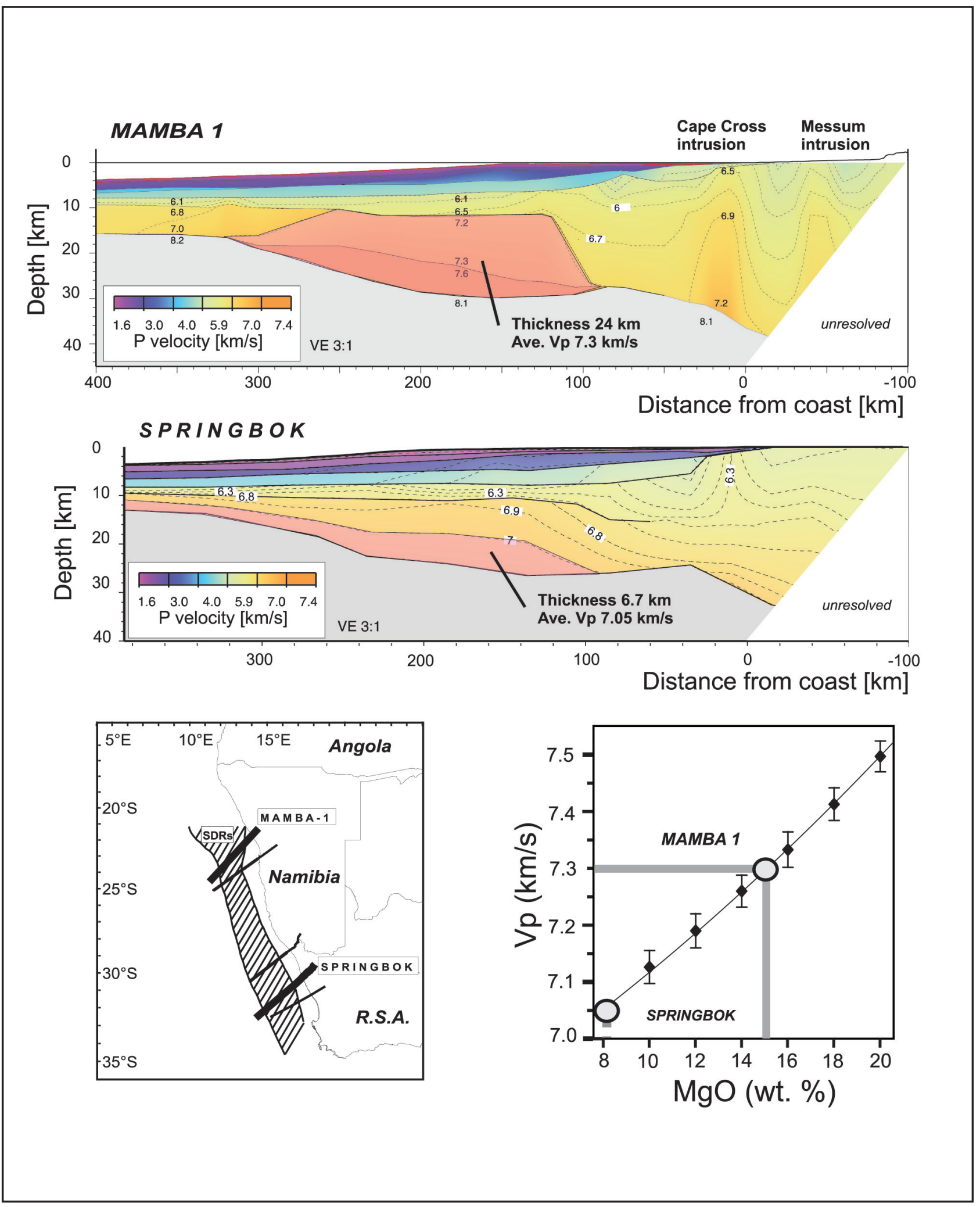

Figure 14. P-wave velocity profiles for two onshore-offshore seismic traverses closest to the areas of HOD and False Bay dike swarms (see location map). The diagonally-ruled field on the location map represents the seaward-dipping reflector sequences (SDRs, from Gladzencko et al., 1997). The MAMBA-1 profile (Bauer et al. 2000) corresponds directly to the HOD area. The steep velocity anomalies in continental crust at the right of the profile are caused by gabbroic roots of the Messum and Cape Cross intrusions (Bauer et al., 2003). The SPRINGBOK profile (Mahanyele et al., 2004) lies north of the False Bay swarm but is currently the only source of velocity information for the low-flux margin segment. High-velocity lower crustal bodies are found in both profiles (outlined in pink) but their thickness and average velocity are both higher in the north. Petrophysical models summarized in the lower right plot (modified from Trumbull et al. 2002) show that the average seismic velocities of lower crust at the northern and southern profiles correspond to gabbros with about 15 and 8 weight $\% \mathrm{MgO}$, respectively, in good agreement with the composition of dolerite dikes from the respective regions. See text for discussion. 
is done, a contrast in $\mathrm{Sr}$ initial ratios between the False Bay and HOD Tafelberg dolerites remains, and this is attributed to differences in the mantle source. Note that for $\mathrm{Pb}$ isotopes (Figure 11), crustal assimilation is still evident in the restricted sample set because of the large contrast in $\mathrm{Pb}$ concentration in primitive basalts vs. continental crust. However, in this case, too, the comparison of $\mathrm{Pb}$ isotope ratios and trace element concentrations ( $\mathrm{Pb} / \mathrm{Ce}$ ratios, Figure 11) allows discrimination of samples with crustal assimilation from those affected by source variations.

For many of the trace elements and their isotopic systems used to distinguish magma types (Figures 9 and 10), variations within the Mg-restricted sample set can be mostly attributed to source heterogeneity in the lithospheric and sub-lithospheric mantle. A diagram commonly used to distinguish different mantle and crustal contributions in igneous suites is shown in Figure 13 with our data from west coast dolerite dikes (>6 weight $\% \mathrm{MgO}$ and $<0.2 \mathrm{Rb} / \mathrm{Sr}$ ), and other components of the Early Cretaceous Paraná-Etendeka LIP. In this figure, the MORB composition lies at one end of the gray field labeled "mantle array" and the Tristan OIB and inferred plume-related alkaline complexes from Namibia plot at the other end. The False Bay dolerites and the HOD Horingbaai types plot within the mantle array, closer to the MORB composition than to the plume/Tristan OIB, which is consistent with their depleted Sr-Nd isotope ratios. Nepheline-normative HOD dolerites tend to plot at mid-range within the mantle array, except for one sample (326), which overlaps with "plume-related" Tristan OIB and alkaline complexes. Some Tafelberg-type HOD dikes overlap with Horingbaai types at mid-range in the mantle array and others trend outside it towards the higher $\mathrm{Th} / \mathrm{Yb}$ and lower $\mathrm{Nb} / \mathrm{Yb}$ ratios typical of the low-Ti CFB field. Crustal assimilation could cause this trend (as well as the high $\mathrm{Sr}$ and low Nd isotope ratios in Tafelberg dikes, see Figure 10), but the fact that all samples plotted on Figure 13 have similar high $\mathrm{MgO}$ and low $\mathrm{Rb} / \mathrm{Sr}$ makes this explanation unlikely. Instead, we suggest that the particular compositions of Tafelberg-dikes, like their CFB counterparts (e.g., Hawkesworth et al., 1999), are features of the continental lithospheric mantle. If so, the distinction of Tafelberg and Horingbaai-types is readily explained by different proportions of asthenospheric vs. lithospheric magma sources in the two magma types (Peate and Hawkesworth, 1996). Turning to the False Bay magmas, their position in Figures 10 and 13 relative to the Tafelberg dikes and CFB can be explained by more asthenospheric input at False Bay, or by a different composition and/or age structure of the subcontinental lithospheric mantle under the Saldanha Belt (Cape Peninsula) vs. the central Damara Belt. With present data, these alternatives cannot be distinguished.

Picritic magmas and regional differences in mantle temperatures

Dikes in both the northern and southern margin segments cover a considerable range of $\mathrm{MgO}$ and there is a large region of overlap below 8 weight \% (Figure 6). However, that value is the maximum for any of the southern localities wherease a large number of dolerites from the HOD swarm, including representatives of all magma types, extend to much higher values (see also Thompson et al., 2001). Furthermore, we concluded from CIPW norm projections and olivine compositions that the high $\mathrm{MgO}$ contents are in most cases characteristic of the melts and not due to olivine accumulation. The same arguments hold for the False Bay samples and we believe that they also are reasonably representative of the emplaced magmas. The discovery of xenocrystic olivines with $\mathrm{FO}_{93}$ in some Horingbaai dikes led Thompson and Gibson (2000) to propose that unerupted Mg-rich magma lay beneath coastal Namibia. The possibility that more Mg-rich rocks also lie hidden beneath the False Bay dike swarm cannot be ruled out but there is no evidence for it in the rocks and we conclude that the contrast in $\mathrm{MgO}$ contents in the two dike swarms is a true feature of the primary melts generated in the respective margin segments. The implication of this is that not only was magma production higher in the northern margin segment than in the south, but also the temperatures of melt generation and the degree of melting. This is obviously relevant to the currently debated role of a thermal mantle plume in producing the Paraná-Etendeka LIP.

Independent evidence for a contrasting composition of melts produced at the high-flux vs. low-flux margin segments comes from a comparison of seismic velocities in the lower crust. Five wide-angle seismic experiments have been conducted across the margin from Namibia to the Cape Province (Figure 14). Seismic velocity profiles for the two northern traverses (Bauer et al., 2000; Trumbull et al., 2002) reveal thick, high-velocity bodies at the continent-ocean transition (COT). A full interpretation of the 3 traverses in South Africa is pending and the only available velocity profile is from the SPRINGBOK traverse (Mahanyele et al., 2004). The MAMBA-1 profile indicates a high-velocity lower crust up to $20 \mathrm{~km}$-thick, with average $\mathrm{P}$-wave velocity = $7.3 \mathrm{~km} / \mathrm{s}$ (Figure 14), whereas the SPRINGBOK profile shows a much thinner $(\sim 7 \mathrm{~km})$ lower crustal body with average P-wave velocity of $7.05 \mathrm{~km} / \mathrm{s}$. Trumbull et al. (2002) attributed the high-velocity lower crust in the MAMBA profiles to gabbroic intrusions and they inferred from petrophysical modelling that the average seismic velocity requires a bulk composition of about 15 weight $\% \mathrm{MgO}$ (lower-right plot, Figure 14). The same seismic velocity vs. composition model applied to the SPRINGBOK profile with $\mathrm{Vp}=7.05$, would predict an $\mathrm{MgO}$ composition of 8 weight $\%$ for the intruded magmas. These petrophysical estimates for about $15 \%$ vs. 8 weight $\% \mathrm{MgO}$ in lower crustal intrusions beneath the high-flux and low-flux margins, respectively, are in good agreement with the contrasting $\mathrm{MgO}$ contents of dolerites from the HOD and False Bay swarms. One can go one step further and estimate 
mantle potential temperatures that would be consistent with these contrasting melt compositions Thompson et al. (2001) used the approach of Langmuir et al. (1992) to derive a mantle potential temperature of $1560^{\circ} \mathrm{C}$ and $20 \%$ degree of melting from an inferred 15 weight \% $\mathrm{MgO}$ for parental melts of the Horingbaai-type dolerites. Trumbull et al. (2002) calculated essentially the same value for potential temperature based on modeling the lower crustal seismic velocities from the MAMBA profiles. A higher potential temperature of about $1700^{\circ} \mathrm{C}$ was inferred by Thompson and Gibson (2000) based on calculations of a melt composition (24 weight \% $\mathrm{MgO}$ ) that could precipitate $\mathrm{FO}_{93}$ olivine xenocrysts found in Horingbaai-type dikes. We did not encounter such high$\mathrm{Mg}$ grains in our samples of the HOD dikes, but olivines with $\mathrm{FO}_{90}{ }^{-93}$ were confirmed in Tafelkop-type lavas and picritic dikes in the Huab area (authors' unpublished data and D. Jerram, personal communication, 2006). The 8 weight $\% \mathrm{MgO}$ for False Bay dolerites and the lower crustal Vp value of $7.05 \mathrm{~km} / \mathrm{s}$ at the SPRINGBOK profile are similar to the corresponding values for oceanfloor basalts, and we note that the lower crustal body at the COT on the SPRINGBOK profile is continuous with oceanic layer 3 at the left end of the profile (Figure 14). Thus, for the southern segment of the margin, we infer a mantle potential temperature of about $1300^{\circ} \mathrm{C}$ by analogy to conditions for MORB generation (Langmuir et al., 1992).

\section{Conclusions}

The results of this study outline important differences in the composition of contemporary dolerite dikes emplaced along the Atlantic margin in Namibia and South Africa. The False Bay dolerites from the Cape Peninsula in the south comprise a single magma series of the low-Ti tholeiitic type. Their trace element abundance patterns and $\mathrm{Sr}-\mathrm{Nd}-\mathrm{Pb}$ isotope ratios, after correction for locally strong crustal contamination, suggest derivation from a lithospheric mantle source or a mixture of enriched lithosphere and asthenospheric sources. In the northern, high-flux margin segment, dolerite dikes from the HOD swarm in Namibia comprise at least three magma types. The Horingbaai type has trace element and isotopic compositions suggesting a MORB-like asthenospheric source. The low-Ti tholeiitic Tafelberg type, which has enriched isotopic and trace element signatures like the Etendeka CFB magmas, was derived largely from an enriched lithospheric mantle source with some crustal input. The nepheline-normative alkaline type has variable isotopic and trace element compositions which are suggestive for contributions from both depleted and enriched mantle sources. None of the dolerite dikes studied had compositional signatures consistent with direct derivation from the Tristan mantle plume, with the possible exception of alkaline dike, sample 326, which occurs near one of the central intrusive complexes. Picritic bulk compositions with up to 16 weight $\% \mathrm{MgO}$ and olivines with up to $\mathrm{FO}_{90}$ are not uncommon in the
HOD swarm whereas dolerites from False Bay and other southern localities do not exceeed 8 weight $\% \mathrm{MgO}$ in whole rock and $\mathrm{FO}_{70}$ in olivines.

The contrast in magmatic intensity in the northern vs. the southern margin segments and the difference in $\mathrm{MgO}$ for dolerite dikes indicate a gradient in both volumes and temperatures of breakup-related magmatism. This is consistent with observed north to south contrasts in the thickness $(\sim 20$ versus $7 \mathrm{~km})$ and average P-wave velocity ( 7.3 versus $7.05 \mathrm{~km} / \mathrm{s}$ ) of the high-velocity lower crust at the continent-ocean transition. However, the width and thickness of seaward-dipping reflectors along the margin, which presumably reflect the volumes of magma extruded near the developing rift, are nearly constant from north to south. Evidence from seafloor-spreading anomalies and age of synrift basins suggest that the south Atlantic opening proceeded from south to north (Nürnberg and Müller, 1991; Gladczenko et al., 1997). In the northern segment the propagating rift encountered a region influenced by a major mantle plume which added heat to the system. This greatly enhanced the magma production in the north, but mechanical limits on accumulated lava thickness imposed by degree of subsidence and magma pressure means the SDRs in the north are no thicker than in the south, just that lavas spread well beyond the initial rift valley to cover the Paraná Basin and much of what is now coastal Namibia.

\section{Acknowledgements}

This work has been motivated and supported by funds made available by the GeoForschungsZentrum Potsdam and University of Cape Town in the framework of the INKABA yeAFRICA program. DLR is grateful to the GFZ Potsdam for supporting his stay as visiting scientist during which this manuscript was prepared. We are grateful for analytical support by Kathrin Schulz, Sabine Tonn, Rudolf Naumann and Heike Rothe (GFZ Potsdam). Finally, we thank Dougal Jerram and Martin Klausen for thorough and constructive reviews that led to great improvements to the paper. This is Inkaba yeAfrica contribution number 18 .

\section{References}

Allsopp, H.L. and Kolbe. P. (1965). Isotopic age determinations on the Cape Granite and intruded Malmesbury sediments, Cape Peninsula, South Africa. Geochimica et Cosmochimica Acta, 29, 1115-1130.

Bauer, K., Neben, S., Schreckenberger, B., Emmermann, R., Hinz, K., Fechner, N., Gohl, K., Schulze, A., Trumbull, R.B. and Weber, K. (2000). Deep structure of the Namibia continental margin as derived from integrated geophysical studies, Journal of Geophysical Research, 105, 25829-25853.

Bauer, K., Trumbull, R.B. and Vietor, T. (2003). Geophysical images and a crustal model of intrusive structures beneath the Messum ring complex, Namibian rifted margin. Earth and Planetary Science Letters, 216, 65-80.

Chevallier, L. and Woodford, A. (1999). Morpho-tectonics and mechanism of emplacement of the dolerite rings and sills of the western Karoo, South Africa. South African Journal of Geology, 102, 43-54.

Corner, B. (2000). Crustal framework of Namibia derived from magnetic and gravity data. Communications of the Geological Survey Namibia, 12, 13-19. Corner, B. (2002). Structural mapping of Namibia derived from interpretation of geophysical and geological data. 11th IAGOD Quadrennial Symposium 
and Geocongress, 22-26 July, 2002, Windhoek, Namibia. Extended Abstract Volume (CD), Geological Survey of Namibia, unpaginaged (CD).

Cox, K.G. (1988). The Karoo Province. In: J. D. Macdougall (Editor), Continental Flood Basalts. Kluwer Academic, Dordrecht, The Netherlands, 239-271.

Da Silva, L.C., Gresse, P.G., Scheepers, R., McNaughton, N.J., Hartmann, L.A and Fletcher, I. (2000). U-Pb SHRIMP and Sm-Nd age constraints on the timing and sources of the Pan-African Cape Granite Suite, South Africa. Journal of African Earth Sciences, 30, 795-815.

Day, R.W. (1987). False Bay dolerites. Annals Geological Survey of South Africa, 21, 1-7.

de Beer, C.H., Gresse, P.G., Theron, J.N. and Almond, J.E. (2002). The Geology of the Calvinia Area. Explanation of 1:250,000 scale Sheet 3118 Calvinia. The Council for Geoscience South Africa.

De Villiers, J. and Sohnge, P.G. (1959). The geology of the Richtersveld. Memoir of the Geological Survey South Africa, 48, 295pp.

de Wit, M. and Horsfield, B. (2006). Inkaba yeAfrica Project Surveys Sector of Earth from Core to Space, EOS Transactions American Geophysical Union, 87, 113.

Dulski, P. (2001). Reference materials for geochemical studies: New analytical data by ICP-MS and critical discussion of reference values. Geostandards Newsletter, 87-125.

Duncan, A.R., Armstrong, R.A., Erlank, A.J., Marsh, J.S. and Watkins, R.T. (1990). MORB-related dolerites associated with the final phases of Karoo flood basalt volcanism in southern Africa. In: J.A.J. Parker, P. C. Rickwood and D.H. Tucker (Editors), Mafic Dikes and Emplacement Mechanisms, A.A. Balkema, Rotterdam, The Netherlands, 119-129.

Duncan, A.R., Erlank, A.J., and Marsh, J.S. (1984). Regional geochemistry of the Karoo Igneous Province. In: A.J. Erlank, (Editor), Petrogenesis of Volcanic Rocks of the Karoo Province. Special Publication of the Geological Society of South Africa, 13, 355-388.

Duncan, R.A., Hargraves, A. and Brey, G.P. (1978). Age, paleomagnetism and chemistry of melilite basalts in the Southern Cape, South Africa. Geological Magazine, 115, 317-327.

Duncan, R.A., Hooper, P.R., Rehacek, J., Marsh, J.S. and Duncan, A.R. (1997). The timing and duration of the Karoo igneous event, southern Gondwana. Journal of Geophysical Research, 102, 18127-18138.

Eales, H.V., Marsh, J.S. and Cox, K.G. (1984). The Karoo igneous province: an introduction. In: A. J. Erlank (Editor), Petrogenesis of Volcanic Rocks of the Karoo Province. Special Publication of the Geological Society of South Africa, 13, 1-26.

Eberle, D.G., Andritzky, G., Hutchins, D.G. and Wackerle, R. (2002) The regional magnetic data set of Namibia: Compilation, contributions to crustal studies and support to natural resource management. South African Journal of Geology, 105, 361-380.

Erlank, A.J., Marsh, J.S., Duncan, A.R., Miller, R.M., Hawkesworth, C.J., Betton, P.J. and Rex, D.C. (1984). Geochemistry and petrogenesis of the Etendeka volcanic rocks from SWA/ Namibia. In: A.J. Erlank (Editor), Petrogenesis of Volcanic Rocks of the Karoo Province. Special Publication of the Geological Society of South Africa, 13, 195-245.

Ewart A., Milner, S.C., Armstrong, R.A., Duncan, A.R. (1998). Etendeka volcanism of the Goboboseb Mountains and Messum Igneous Complex, Namibia. Part I: Geochemical evidence of early Cretaceous Tristan plume melts and the role of crustal contamination in the Parana-Etendeka CFB. Journal of Petrology, 39, 191-225.

Ewart, A., Marsh, J.S., Milner, S.C., Duncan, A.R., Kamber, B.S., Armstrong, R.A. (2004). Petrology and geochemistry of Early Cretaceous bimodal continental flood volcanism of the northwest Etendeka, Namibia. Part 1 Introduction, mafic lavas and re-evaluation of mantle source components. Journal of Petrology, 45, 59-105.

Frimmel, H.E. and Frank, W. (1998). Neoproterozoic tectonothermal evolution of the Gariep Belt and its basement, Namibia and South Africa. Precambrian Research, 90, 1-28.

Gerstenberger, H. and Haase, G. (1997). A highly effective emitter substance for mass spectrometric $\mathrm{Pb}$ isotope ratio determination. Chemical Geology, 136, 309-312.

Gibson, S.A., (2002). Major element heterogeneity in Archean to Recent mantle plume starting-heads. Earth and Planetary Science Letters, 195, 5974.

Gibson S.A., Thompson, R.N., Weska, R.K., Dickin, A.P. and Leonardos, O.H. (1997). Late-Cretaceous rift-related upwelling and melting of the Trinidade starting mantle plume head beneath western Brazil. Contributions to Mineralogy and Petrology, 126, 303-314.

Gladczenko, T.P., Hinz, K., Eldholm, O., Meyer, H., Neben, S. and Skogseid, J. (1997). South Atlantic volcanic margins. Journal Geological Society London, 154, 465-470.

Haack, U., Hoefs, J. and Gohn, E. (1982). Constraints on the origin of Damaran granites by $\mathrm{Rb} / \mathrm{Sr}$ and delta ${ }^{18} \mathrm{O}$ data. Contributions to Mineralogy and Petrology, 79, 279-289.

Hahne, K. (2004) Detektierung eines mesozoischen Gangschwarmes in northwest Namibia und Rekonstruktion regionaler Spannungszustände während der Südatlantiköffnung. Unpublished Ph.D. dissertation, University of Potsdam, Germany, 125 pp (in German).

Harris, C. (1995). Oxygen isotope geochemistry of the Mesozoic anorogenic complexes of Damaraland, northwest Namibia: evidence for crustal contamination and its effect on silica saturation. Contributions to Mineralogy and Petrology, 122, 308-321.

Harris, C., Marsh, J.S., Milner, S.C., (1999). Petrology of the alkaline core of the Messum igneous complex, Namibia: evidence for the progressively decreasing effect of crustal contamination. Journal of Petrology 40, 1377-1397.

Hart, S.R. (1988). Heterogeneous mantle domains: signatures, genesis and mixing chronologies. Earth and Planetary Science Letters 90, 272-296.

Hawkesworth, C.J., Kelley, S., Turner, S., le Roex, A. and Storey, B. (1999). Mantle processes during Gondwana break-up and dispersal. Journal of African Earth Science, 28, 239-261.

Hinz, K. (1981). A hypothesis on terrestrial catastrophes; wedges of very thick oceanward dipping layers beneath passive continental margins; their origin and paleoenvironmental significance. Geologisches Jahrbuch. Reibe E: Geophysik. 22, 28pp.

Hinz, K., Neben, S., Schreckenberger, B., Roeser, H.A., Block, M., Goncalves de Souza, K. and Meyer, H. (1999). The Argentine continental margin north of $48^{\circ} \mathrm{S}$ : Sedimentary successions, volcanic activity during breakup. Marine and Petroleum Geology, 16, 1-25.

Hoffmann, A.W. (1997). Mantle geochemistry: the message from oceanic volcanism. Nature, 385, 219-229.

Hunter, D.R. and Reid, D.L. (1987). Mafic dike swarms in Southern Africa. In: H. C. Halls and W. F. Fahrig (Editors), Mafic Dike Swarms. Geological Association of Canada Special Paper, 34, 445-456.

Jaffey, A.H., Flynn, K.F., Glendenin, L.E., Bentley, W,C. and Essling, A.M. (1971). Precision measurement of half-lives and specific activities of ${ }^{235} \mathrm{U}$ and ${ }^{238}$ U. Physics Reviews, C4, 1889-1906.

Janney, P.E., le Roex, A.P., Carlson, R.W. and Viljoen, K.S. (2002). A chemical and multi-isotope study of the Western Cape olivine melilite province, South Africa: Implications for the sources of kimberlites and the origin of the HIMU signature in Africa. Journal of Petrology, 43, 2339-2370.

Jansen, H. (1960). The geology of the Bitterfontein Area, Cape Province. An explanation of sheet 253 (Bitterfontein). Geological Survey South Africa. $97 \mathrm{pp}$.

Jerram, D., Mountney, N., Holzförster, F. and Stollhofen, H. (1999). Internal stratigraphic relationships in the Etendeka Group in the Huab Basin, north west Namibia: understanding the onset of flood volcanism. Journal of Geodynamics, 28, 393-418.

Jung, S., Hoernes, S. and Mezger, K., (2000). Geochronology and petrogenesis of Pan-African, syn-tectonic, S-type and post-tectonic A-type granite (Namibia): products of melting of crustal sources, fractional crystallization and wallrock entrainment. Lithos, 50, 259-287.

Kirstein, L.A., Kelley, S., Hawkesworth, C., Turner, S., Mantovani, M. and Wijbrans, J. (2001). Protracted felsic magmatic activity associated with the opening of the South Atlantic. Journal of the Geological Society, London, 158, 583-592.

Kolbe, P. and Taylor, S.R. (1966). Major and trace element relationships in granodiorites and granites from Australia and South Africa. Contributions to Mineralogy and Petrology, 12, 202-222.

Kurszlaukis, S., Franz, L. and Lorenz, V. (1998). On the volcanology of the Gibeon Kimberlite Field, Namibia. Journal of Volcanology and Geothermal Research, 84, 257-272.

Langmuir, C.H., Klein, E., and Plank, T. (1992). Petrological systematics of mid-ocean ridge basalts: constraints on melt generation beneath ocean ridges. In: J. Phipps Morgan, D. K. Blackman and J. M. Sinton (Editors), Mantle Flow and Melt Generation at Mid-Ocean Ridges, American Geophysical Union, Geophysical Monograph 71, 183-280. 
Mahanyele, P.J., Bauer, K., Franke, D., Schulze, A., Ryberg, T., de Beer, C.H., Neben, S., Schreckenberger, B. and Stettler, E.H. (2004). How far to the south does the volcanic margin of Southwest Africa extend? An initial velocity model for the ocean-continent transition in the southern Cape Basin. 64. Jahrestagung der Deutschen Geophysikalischen Gesellschaft, Berlin, 8-12 March, 2004. Abstract volume, 443-444.

Marais, J.A.H., Agenbacht, A.L.D., Prinsloo, M. and Basson,W.A. (2002). The geology of the Springbok area. Sheet 2917. Springbok, Pretoria, Council for Geoscience, South Africa, scale $1: 250,000$.

Marsh, J.S. (1975). The Luderitz alkaline province, south west Africa I: Descriptive petrology of the Granitberg foyaite complex. Transactions Geological Society South Africa, 78, 215-224.

Marsh, J.S. (1976). The Luderitz alkaline province, south west Africa III: The Pomona and Drachenberg syenite complexes. Transactions Geological Society South Africa, 79, 168-176.

Marsh, J.S., Hooper, P.R., Rehacek, J., Duncan, R.A. and Duncan, A.R. (1997) Stratigraphy and age of the Karoo basalts of Lesotho and implications for correlations within the Karoo Province. In: J.J., Mahoney, M.E. Coffin (Editors.) Large Igneous Provinces; Continental, Oceanic, and Planetary Flood Volcanism. American Geophysical Union, Geophysical Monographs, 100, 247-272.

Marsh, J.S., Ewart, A., Milner, S.C., Duncan, A.R. and Miller, R. McG. (2001) The Etendeka Igneous Province: magma types and their stratigraphic distribution with implications for the evolution of the Paraná-Etendeka flood basalt province. Bulletin of Volcanology 62, 464-486.

Marzoli, A., Melluso, L., Morra, V., Renne, P.R., Sgrosso, I., D’Antonio, M., Duarte Morais, L., Morais, E.A.A. and Ricci, G. (1999). Geochronology and petrology of Cretaceous basaltic magmatism in the Kwanza basin (western Angola), and relationships with the Paraná-Etendeka continental flood basalt province. Journal of Geodynamics, 28, 341-356.

McDermott, F., Harris, N.B.W. and Hawkesworth, C.J. (1996). Geochemical constraints on crustal anatexis: a case study from the Pan-African Damara granitoids of Namibia. Contributions to Mineralogy and Petrology, 123, 406-423.

McIver, J.R. (1981). Aspects of ultrabasic and basic alkaline intrusive rocks from Bitterfontein, South Africa. Contributions to Mineralogy and Petrology, 78, 1-11

Miller, R.McG. (1983). The Pan-African Damara Orogen of South West Africa/Namibia. In: R. McG. Miller (Editor), Evolution of the Damara Orogen of South West Africa/Namibia. Geological Society of South Africa Special Publication, 11, 431-515.

Milner S.C. and le Roex, A.P. (1996). Isotope characteristics of the Okenyenya igneous complex northwestern Namibia: constraints on the composition of the early Tristan plume and the origin of the EM1 mantle component. Earth and Planetary Science Letters, 141, 277-291.

Mohriak, W.U., Rosendahl, B.R., Turner, J.P. and Valente, S.C. (2002). Crustal architecture of South Atlantic volcanic margins, In: M.A. Menzies, S.L. Klemperer, C.J. Ebinger and J. Baker (Editors), Volcanic rifted margins. Geological Society of America Special Paper, 362, 159-202.

Moore, A.E. (1976). Controls of post-Gondwanaland alkaline volcanism in Southern Africa. Earth and Planetary Science Letters, 31, 291-296.

Moore, A.E. and Verwoerd, W.J. (1985). The olivine melilitite-"kimberlite"carbonatite suite of Namaqualand and Bushmanland, South Africa. Transactions of the Geological Society of South Africa, 88, 281-294.

Morbidelli, L., Gomes, C.B., Beccaluva, L., Brotzu, P., Conte, A.M., Ruberti, E. and Traversa, G. (1995). Mineralogical, petrological and geochemical aspects of alkaline and alkaline-carbonatite associations from Brazil. Earth Science Reviews, 39, 135-168.

Nürnberg, D. and Müller, R. (1991). The tectonic evolution of the South Atlantic from the Late Jurassic to present. Tectonophysics, 191, 27-53.

O'Connor J.M. and le Roex A.P. (1992). South Atlantic hot spot-plume systems: a distribution of volcanism in time and space. Earth and Planetary Science Letters, 113, 343-364.

Peate, D.W. and Hawkesworth, C. (1996). Lithospheric to asthenospheric transition in low-Ti flood basalts from southern Paraná, Brazil. Chemical Geology, 127, 1-24.

Peate, D.W. (1997). The Parana-Etendeka Province. In: Mahoney, J.J., Coffin, M.E. (Eds.) Large Igneous Provinces; Continental, Oceanic, and Planetary Flood Volcanism. American Geophysical Union, Geophysical Monographs, 100, 217-245.

Reeves, C.V. (2000). The geophysical mapping of Mesozoic dike swarms in southern Africa and their origin in the disruption of Gondwana. Journal of African Earth Sciences, 30, 499-513.

Reid, D.L. (1990). The Cape Peninsula dolerite dike swarm, South Africa. In: A. J. Parker, P. C. Rickwood and D. H. Turner (Editors), Mafic Dikes and Emplacement Mechanisms. A. A. Balkema, Rotterdam, The Netherlands, 325-334.

Reid, D.L., Erlank, A.J. and Rex, D.C. (1991). Age and correlation of the False Bay dolerite dike swarm, south-western Cape, Cape Province. South African Journal Geology, 94, 155-158.

Reid, D.L. and Rex, D.C. (1994). Cretaceous dikes associated with the opening of the South Atlantic: the Mehlberg dike, northern Richtersveld. South African Journal of Geology, 97, 135-145.

Reid, D.L., Cooper, A.F., Rex, D.C. and Harmer, R.E. (1990). Timing of postKaroo alkaline volcanism in southern Namibia. Geological Magazine, 127, 427-433.

Renne, P.R., Deckart, K., Ernesto, M., Féraud, G., and Picirillo, E.M. (1996a). Age of Ponta Grossa Dike Swarm (Brazil), and implications to Paraná flood volcanism. Earth and Planetary Science Letters, 144, 199-211.

Renne, P.R., Glen, J.M., Milner, S.C. and Duncan, A.R. (1996b). Age of Etendeka flood volcanism and associated intrusions in southwestern Africa. Geology, 24, 659-662.

Romer, R.L., Heinrich W., Schröder-Smeibidl, B., Meixner, A., Fischer, C.-O. and Schulz, C. (2005) Elemental dispersion and stable isotope fractionation during reactive fluid-flow and fluid immiscibility in the Bufa del Diente aureole, northeast Mexico: Evidence from radiographies and Li, B, Sr, Nd, and $\mathrm{Pb}$ isotope systematics. Contribution to Mineralogy and Petrology, 149, 400-429.

Rozendaal, A., Gresse, P.G., Scheepers, R. and le Roux, J.P. (1999). Neoproterozoic to Early Cambrian crustal evolution of the Pan-African Saldanha Belt, South Africa. Precambrian Research, 97, 303-323.

Saunders, A.D., Fitton, J.G., Kerr, A.C., Norry, M.J. and Kent, R.W. (1997). The North Atlantic Igneous province. In: J.J. Mahoney, M.E. Coffin, (Editors.) Large Igneous Provinces; Continental, Oceanic, and Planetary Flood Volcanism. American Geophysical Union, Geophysical Monographs, 100, 45-93.

Schmitt, A.K., Emmermann, R., Trumbull, R.B., Bühn, B. and Henjes-Kunst, F. (2000). Petrogenesis and ${ }^{40} \mathrm{Ar} /{ }^{39} \mathrm{Ar}$ Geochronology of the Brandberg complex, Namibia: Evidence for a major mantle contribution in metaluminous and peralkaline granites. Journal of Petrology, 41, 1207-1239.

Siebel, W., Becchio, R., Volker, F., Hansen, M.A.F., Viramonte, J., Trumbull, R.B., Haase, G. and Zimmer, M. (2000). Trinidade and Martin Vaz islands, South Atlantic: isotopic $(\mathrm{Sr}, \mathrm{Nd}, \mathrm{Pb})$ and trace element constraints on plume related magmatism. Journal of South American Earth Sciences, 13, 79-103. Siedner, G. and Mitchell, J.G. (1976). Episodic Mesozoic volcanism in Namibia and Brazil; K-Ar isochron study bearing on the opening of the South Atlantic. Earth and Planetary Science Letters, 30, 292-302.

Stewart, K., Turner, S., Kelley, S., Hawkesworth, C., Kirstein, L. and Mantovani, M. (1996). 3-D ${ }^{40} \mathrm{Ar}^{-39} \mathrm{Ar}$ geochronology in the Paraná continental flood basalt province. Earth and Planetary Science Letters, 143, 95-109.

Steiger, R.H. and Jäger, E. (1977). Subcommission of geochronology: Convention on the use of decay constants in geo- and cosmochronology. Earth and Planetary Science Letters, 36, 359-362.

Sun, S.S. and McDonough, W.F. (1989). Chemical and isotopic systematics of oceanic basalts: implications for mantle composition and processes. Geological Society of London Special Publication, 42, 313-345.

Tankard, A.J., Jackson, M.P.A., Eriksson, K.A., Hobday, D.K., Hunter, D.R. and Minter, W.E.L. (1982). Crustal Evolution of South Africa; 3.8 Billion Years of Earth History. Springer, New York, United States of America, 523pp. Thompson, R.N. and Gibson, S.A. (2000). Transient high temperatures in mantle plume heads inferred from magnesian olivines in Phanerozoic picrites. Nature, $\mathbf{4 0 7}, 502-506$.

Thompson, R.N., Gibson, S.A., Dickin, A.P. and Smith, D.P. (2001). Early Cretaceous basalt and picrite dikes of the southern Etendeka region, northwest Namibia: windows into the role of the Tristan mantle plume in Paraná-Etendeka magmatism. Journal of Petrology, 42, 2049-2081.

Thompson, R.N., Morrison, M.A., Dickin, A.P. and Hendry, G.L. (1983). Continental flood basalts ... Arachnid's rule OK. In: C. J. Hawkesworth and M. J. Norry (Editors), Continental Flood Basalts and Mantle Xenoliths. Shiva, Nantwich, United Kingdom, 158-185. 
Thompson, R.N., Riches, A.J.V., Antoshechkina, P.M., Pearson, D.G., Nowell, G.M., Ottley, C.J., Dickin, A.P., Hards, V.L., Nguno, A.-K. and Niku-Paavola, V. (2007). Origin of CFB magmatism: multi-tiered intracrustal picriterhyolite magmatic plumbing at Spitzkoppe, western Namibia, during Early Cretaceous Etendeka magmatism. Journal of Petrology, 48, 1119-1154

Trumbull, R.B., Bühn, B., Romer, R.L and Volker, F. (2003). The petrology of basanite-tephrite intrusions in the Erongo complex and implications for a plume source of Cretaceous alkaline complexes in Namibia. Journal of Petrology, 44, 93-112.

Trumbull, R.B., Emmermann, R., Bühn, B., Gerstenberger, H., Mingram, B., Schmitt, A. and Volker, F. (2000). Insights on the genesis of the Cretaceous Damaraland igneous complexes in Namibia from a Nd- and Sr-isotopic perspective. Communications of the Geological Survey of Namibia, 12, 313-324

Trumbull, R.B., Harris, C., Frindt, S. and Wigand, M. (2004a). Oxygen and neodymium isotope evidence for source diversity in Cretaceous anorogenic granites from Namibia and implications for A-type granite genesis. Lithos, 73, 21-40.

Trumbull, R.B., Sobolev, S.V. and Bauer, K. (2002). Petrophysical modeling of high seismic velocity crust at the Namibian volcanic margin. In: M.A. Menzies, S.L. Klemperer, C.J. Ebinger and J. Baker (Editors), Volcanic Rifted Margins, Geologic Society of America Special Paper, 362, 221-230.

Trumbull, R.B., Vietor, T., Hahne, K., Wackerle, R. and Ledru, P. (2004b). Aeromagnetic mapping and reconnaissance geochemistry of the Early Cretaceous Henties Bay-Outjo mafic dike swarm, Etendeka Igneous Province, Namibia. Journal of African Earth Sciences, 40, 17-29.

Ulmer, P. (1989). The dependence of the $\mathrm{Fe}^{2+}-\mathrm{Mg}$ cation-partitioning between olivine and basaltic liquid on pressure, temperature and composition. An experimental study to $30 \mathrm{kbars}$. Contributions to Mineralogy and Petrology, 101, 261-273.

Van Acken, D. (2006). Geochemische Untersuchungen an basaltischen Gängen des Hentiesbaai-Outjo-Gangschwarms, Namibia. Unpublished M.Sc. thesis, Free University of Berlin, Germany, 66 pp (in German).

Vietor, T. and Weber, K. (1999). Orientation and density distribution of Cretaceous dikes in central Namibia from high resolution aeromagnetics. European Union of Geosciences conference abstracts; EUG 10. Journal of Conference Abstracts. 4, 1, 347.

Von Veh, M. (1993). The stratigraphy and structural evolution of the late Proterozoic Gariep Belt in the Sendelingsdrif - Annisfontein area, northwestern Cape Province. University of Cape Town, Bulletin of the Precambrian Research Unit, 38, 174pp.

Walker, F. (1956). The dolerites of the Cape Peninsula. Transactions Geological Society South Africa, 77, 77-92.

White, R.S. and McKenzie, D. (1989). Magmatism at continental rift zones: the generation of volcanic continental margins and flood basalts. Journal Geophysical Research, 94, 7865-7729.

Whitehead, K., le Roex, A.P., Class, C. and Bell, D.R. (2002). Composition and Cretaceous thermal structure of the upper mantle beneath the Damara Mobile Belt: evidence from nephelinite-hosted peridotite xenoliths, Swakopmund, Namibia. Journal of the Geological Society, London, 159, 307-321.

Wigand, M., Schmitt, A.K., Trumbull, R.B., Villa, I.M. and Emmermann, R. (2004). Short-lived magmatic activity in an anorogenic subvolcanic complex: ${ }^{40} \mathrm{Ar}^{-39} \mathrm{Ar}$ and ion microprobe U-Pb zircon dating of the Erongo, Damaraland Province, Namibia. Journal of Volcanology and Geothermal Research 130, 285-305.

Editorial handling: M. J. de Wit and Brian Horsfield

\section{Appendix. Analytical methods}

All trace element analyses of the HOD samples and some samples from the False Bay swarm (as designated on Table 2) were determined by inductively coupled plasma mass spectrometry (ICP-MS) at the GFZ Potsdam using a Perkin Elmer ELAN 5000A, following analytical procedures described by Dulski (2001). Additional samples of the False Bay dikes and dikes from the western Cape localities (Cedarberg, Garies, Saldanha Bay) were analyzed by ICP-MS at the University of Cape Town.

Radiogenic $\mathrm{Sr}, \mathrm{Nd}$ and $\mathrm{Pb}$ isotope analyses were made at the GFZ Potsdam on unspiked whole-rock powders. Samples were disssolved with $42 \% \mathrm{HF}$ for four days at $160^{\circ} \mathrm{C}$ on the hot plate. Digested samples were dried and taken up in $6 \mathrm{~N}$ HCL. Pb was separated using $\mathrm{HBr}-\mathrm{HCl}$ ion-exchange and $\mathrm{Sr}$ and $\mathrm{Nd}$ were separated and purified using ion-exchange chromatography as described in Romer et al. (2005). Strontium was loaded on single Ta filaments and isotopic composition determined on a VG Sector 54-30 multi-collector mass spectrometer using triple-jump dynamic multi-collection. Ratios of ${ }^{86} \mathrm{Sr} /{ }^{88} \mathrm{Sr}$ and ${ }^{143} \mathrm{Nd} /{ }^{144} \mathrm{Nd}$ were normalized to values of 0.1194 and 0.7219 , respectively. Analytical uncertainties are given at the $2 \sigma_{\mathrm{m}}$ level. Repeated analyses of Sr reference material NBS 987 during the measurement period yielded ${ }^{87} \mathrm{Sr} /{ }^{86} \mathrm{Sr}$ ratios of 0.710249 $\pm 0.000004(2 \sigma$ reproducibility, $n=12)$. Total procedural blanks were $<50 \mathrm{pg}$ Sr. Nd was loaded on double Re-filaments and its isotopic composition determined on a Finnigan MAT 262 multi-collector mass spectrometrer using dynamic multi-collection. Repeated analyses of the La Jolla Nd standard during the measurement period gave ${ }^{143} \mathrm{Nd} /{ }^{144} \mathrm{Nd}=0.511850 \pm 0.000004$ $(2 \sigma$ reproducibility, $n=14)$. The total procedural blank was $<30 \mathrm{pg} \mathrm{Nd}$. Initial $\mathrm{Sr}$ and $\mathrm{Nd}$ ratios were calculated for $\lambda^{87} \mathrm{Rb}=1.42 \mathrm{E}-11 \mathrm{y}^{-1}$ and $\lambda^{147} \mathrm{Sm}=6.54 \mathrm{E}-12 \mathrm{y}^{-1}$, $\left({ }^{147} \mathrm{Sm} /{ }^{144} \mathrm{Nd}\right){ }^{0}{ }_{\mathrm{CHUR}}=0.1967$ and $\left({ }^{143} \mathrm{Nd} /{ }^{144} \mathrm{Nd}\right)^{0}{ }_{\mathrm{CHUR}}$ $=0.512638$, and sample concentration data in Tables 1 and 2. An emplacement age of $130 \mathrm{Ma}$ was assumed for all samples, corresponding to the main event of the regional flood basalt volcanism.

Lead was loaded together with silica-gel and $\mathrm{H}_{3} \mathrm{PO}_{4}$ on single Re filaments (Gerstenberger and Haase, 1997). The isotopic composition was determined at 1200$1250^{\circ} \mathrm{C}$ on a Finnigan MAT 262 multi-collector mass spectrometer using static multi-collector. Instrumental fractionation was corrected with $0.1 \%$ / A.M.U. as determined from repeated measurement of reference material NBS 981. Uncertainties in accuracy and precision of reported $\mathrm{Pb}$ isotope ratios are smaller than $0.1 \%$ at the $2 \sigma$ level. Total procedural blanks are better than $15-30 \mathrm{pg} \mathrm{Pb}$. Initial $\mathrm{Pb}$ isotope ratios calculated for $130 \mathrm{Ma}$ used concentration data in Tables 1 and 2 and constants of Jaffey et al. (1971) recommended by IUGS (Steiger and Jäger, 1977). 